\title{
Assessment of Bulk Absorber Properties for Multi-Layer Perforates in Porous Honeycomb Liners
}

\author{
M. G. Jones* and T. L. Parrott ${ }^{\dagger}$ \\ NASA Langley Research Center, Hampton, VA 23681-2199, USA
}

\begin{abstract}
C YONTINUING progress in materials technology provides potential for improved acoustic liners for attenuating broadband fan noise emissions from aircraft engine nacelles. Conventional liners (local-reacting perforate-over-honeycomb structures) provide significant narrow-band attenuation, but limited attenuation over wide bandwidths. Two approaches for increasing attenuation bandwidth are to (1) replace the honeycomb structure with bulk material, or (2) cascade multiple layers of perforate/honeycomb structures. Usage of the first approach is limited because of mechanical and maintenance reasons, while multi-layer liners are limited to about three layers because of their additional mechanical complexity, depth and weight. The current research concerns a novel approach reported by the University of Cincinnati, in which a single-layer conventional liner is converted into an extended-reaction, broadband absorber by making the honeycomb core structure porous. This modified single-layer liner requires no increase in depth and weight, and minimal increase in mechanical complexity. Langley has initiated research to identify potential benefits of liner structures with porous cell walls. This research has two complementary goals: (1) develop and validate experimental techniques for treating multi-layer perforates (representative of the internal cells of a liner with porous cell walls) as 1-D bulk materials, and (2) develop analytical approaches to validate this "bulk material" assumption. If successful, the resultant model can then be used to design optimized porous honeycomb liners. The feasibility of treating an $N$-layer perforate system ( $N$ porous plates separated by uniform air gaps) as a one-dimensional bulk absorber is assessed using the Two-Thickness Method (TTM), which is commonly used to educe bulk material intrinsic acoustic parameters. Tests are conducted with discrete tone and random noise sources, over an SPL range sufficient to determine the nonlinearity of the test specimens, for test specimens consisting of 5,10 and $15 \%$ porous plates. Measured impedances for two liner thicknesses (e.g., 12 and 24 layers) are used as input to the TTM to determine the characteristic impedance and propagation constant that characterize these liners as bulk absorbers. These parameters are then used to calculate the predicted impedance of liners with different thicknesses (e.g., 36 layers), and a comparison of predicted and measured impedances for these other thicknesses is used to determine the efficacy of this approach. Finally, an independent method is used to educe the propagation constant for a single representative sample, and excellent comparison between the results for this method and those for the TTM provides increased confidence in the results achieved with the TTM. In general, the results demonstrate these multi-layer perforates can be acceptably treated as bulk absorbers.
\end{abstract}

\section{Nomenclature}

$\begin{array}{ll}c & \text { sound speed in air, } \mathrm{m} / \mathrm{s} \\ c_{m} & \text { sound speed in material, } \mathrm{m} / \mathrm{s} \\ d_{1}, d_{2} & \text { depth of thin and thick samples, respectively, used in Two-Thickness Method, } \mathrm{m} \\ d_{s} & \text { sample depth, } \mathrm{m} \\ i & \text { counter, result for } i^{\text {th }} \text { frequency } \\ I & \sqrt{-1} \\ L 2 & \text { L2-Norm } \\ M & \text { number of frequencies used in L2-Norm computation } \\ N & \text { number of layers in multi-layer perforate sample } \\ t & \text { time, s }\end{array}$

* Senior Research Scientist, Research \& Technology Directorate, Structural Acoustics Branch, Mail Stop 463; Michael.G.Jones@ NASA.GOV. Senior member of AIAA.

${ }^{\dagger}$ Senior Research Scientist, Research \& Technology Directorate, Structural Acoustics Branch, Mail Stop 463; Tony.L.Parrott@ NASA.GOV. 
Symbols:

$\begin{array}{ll}\Gamma & =\Gamma_{r}+I \Gamma_{i}, \text { propagation constant, } \mathrm{m}^{-1} \\ \rho & \text { ambient density, } \mathrm{kg} / \mathrm{m}^{3} \\ \theta & \text { acoustic resistance, normalized by } \rho c \\ \omega & \text { angular frequency, } \mathrm{s}^{-1} \\ \chi & \text { acoustic reactance, normalized by } \rho c \\ \zeta_{1} & \text { normal incidence acoustic impedance, normalized by } \rho c, \text { for sample of depth } d \\ \zeta_{2} & \text { normal incidence acoustic impedance, normalized by } \rho c, \text { for sample of depth } 2 d \\ \zeta_{c} & \text { characteristic impedance, normalized by } \rho c \\ \zeta_{m} & \text { measured normal incidence acoustic impedance, normalized by } \rho c \\ \zeta_{p} & \text { predicted normal incidence acoustic impedance, normalized by } \rho c \\ \zeta_{s} & \text { normal incidence acoustic impedance of test sample, normalized by } \rho c \\ \text { Abbreviations: } & \\ \text { OASPL } & \text { overall sound pressure level, dB } \\ \text { SPL } & \text { sound pressure level, dB } \\ \text { TMM } & \text { Two-Microphone Method } \\ \text { TTM } & \text { Two-Thickness Method }\end{array}$

Notes:

Unless specified otherwise, all impedances provided in this paper are normalized by $\rho c$.

The $e^{i \omega t}$ sign convention is used throughout this paper.

\section{Introduction}

$\mathrm{D}$ URING the last few decades, there has been a continuing push toward aircraft engine nacelles with higher bypass ratios. As a result, the emphasis for acoustic liner design has shifted from tonal to broadband. Conventional liners have traditionally consisted of local-reacting, perforate-over-honeycomb structures that provide significant attenuation over a relatively narrow bandwidth. Two approaches for increasing attenuation bandwidth are well known. One approach is to replace the honeycomb structure with bulk materials. ${ }^{1}$ However, for mechanical and maintenance reasons, the usage of bulk materials has been limited. Another approach is to cascade multiple layers of perforate/honeycomb structures $^{2}$ (see figure 1). In practice, these multi-layer liners have been limited to about three layers because of their additional mechanical complexity, depth and weight. Recent tests ${ }^{3}$ with single-layer porous honeycomb liners, in which the honeycomb core is replaced with porous divider plates (see figure 2), demonstrated that an increase in attenuation bandwidth of greater than $70 \%$ can be achieved, but at the expense of peak attenuation reduction by nearly $20 \%$. Perhaps more importantly, the increased attenuation bandwidth was achieved with a modified single-layer liner that required no increase in depth and weight, and required minimal increase in mechanical complexity (porous cell walls).

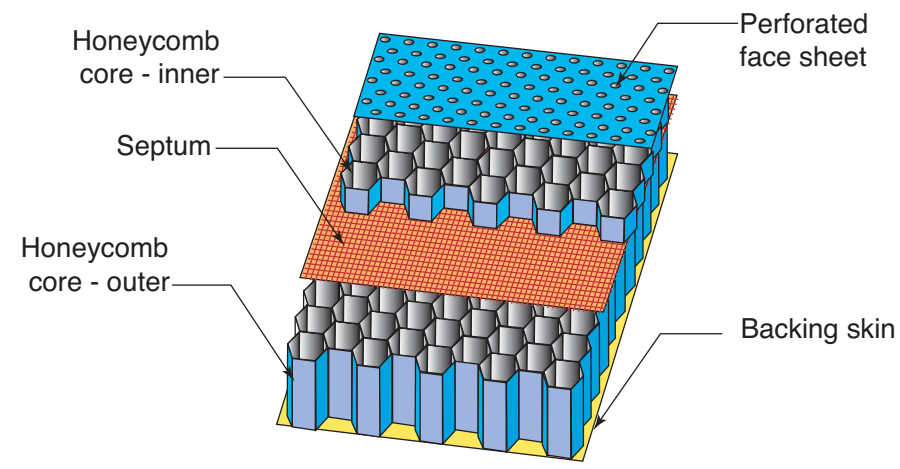

Figure 1. Sketch of conventional multi-layer, perforate-over-honeycomb liner. 
From a liner design point of view, a disadvantage of porous honeycomb liners is the loss of the local-reacting feature of conventional liners. Instead, these structures require more complex liner models that account for their extended-reaction feature. For extended-reaction liners, the impedance boundary condition loses usefulness, and the duct propagation model must couple the liner characteristics with the air passage through the duct. While these extended-reaction liner models are indeed more complicated to develop, they also provide additional "design knobs" that can be used to achieve desired liner properties. These include cell-to-cell spacing and cell wall porosity, each of which can conceivably be varied independently in the axial and circumferential directions within the liner to achieve selected non-isotropic, bulk absorber-like behavior. Therefore, to explore the full potential of this design tool, it is critical that cell-to-cell acoustic coupling via porous cell walls be adequately modeled. It is for this purpose that an experimental and computational program has begun at Langley to modify an existing impedance model such that it is suitable for multi-layer perforates (representative of the internal cells of a liner in which the cell walls are porous). The goal of this research is to use this model, described in a companion paper, ${ }^{4}$ to properly assess the noise reduction capability of porous honeycomb liners.

The first step in this process is to assess the feasibility of treating an $N$-layer perforate system, consisting of $N$ porous plates separated by uniform air gaps, as a one-dimensional bulk absorber. It appears obvious that such a model will be limited in applicability to restricted ranges of $N$, plate porosity and plate-to-plate spacing where the implied "continuum" assumption is satisfied for these honeycomb structures. It is assumed that $N$ should be sufficiently large to allow the bulk acoustic properties of the " $N$-layer liner" to be educed using the Two-Thickness Method ${ }^{5}$ (TTM). This method is used to determine the characteristic impedance and propagation constant spectra (key parameters for description of extended-reaction liners) for homogeneous bulk absorbers. On the other hand, $N$ should be low enough to ensure that sufficient sound can reflect from the liner back-plate. Finally, it is assumed that the plate spacing must be within an acceptable range to ensure that the liner appears homogeneous.

The current investigation was designed to evaluate multi-layer perforates for usage in porous honeycomb acoustic liners. Specifically, this paper will discuss (1) test specimen design, with attention given to linear/nonlinearity issues, (2) usage of the Two-Thickness and Scott methods ${ }^{5,6}$ for multi-layer perforate liner evaluation, and (3) a critique of results for multi-layer perforate liners, with a focus on limits of $N$. Finally, a comparison of "bulk properties" educed for a typical fibrous material (e.g., fiberglass) using the Delany-Bazely semi-empirical model ${ }^{7}$ with those educed for the multi-layer perforate liner using the Two-Thickness Method is presented.

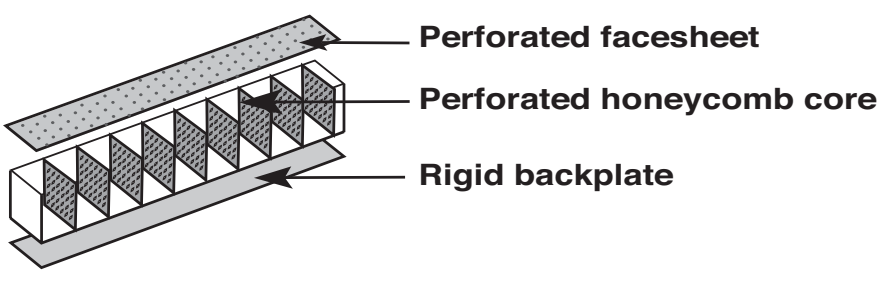

Figure 2. Sketch of porous honeycomb liner.

\section{Test Samples}

DERforated plates (cell divider plates) with porosities of 5, 10 and $15 \%$ were fabricated by means of an electronbeam drilling procedure. Divider plates were fabricated in 55.9- $\mathrm{mm} \times 55.9-\mathrm{mm}$ square samples, with perforations distributed uniformly over the centered $50.8-\mathrm{mm} \times 50.8-\mathrm{mm}$ square section (see figure 3). Each divider plate was fabricated with a thickness of $1.219 \mathrm{~mm}$ and hole diameters of $0.254 \mathrm{~mm}$, to achieve a thickness-to-diameter ratio of 5. Although it is typically assumed that a thickness-to-diameter ratio of 10 is needed to achieve a near-linear liner, it was expected that a ratio of 5 would provide a sufficiently low nonlinear factor such that moderate overall sound pressure levels (up to $120 \mathrm{~dB}$ OASPL) would not cause significant nonlinear response (i.e., no change in absorption properties over a range of incident sound pressure levels). Spacers (see figure 4) are used to achieve a $6.731 \mathrm{~mm}$ centerto-center spacing. These spacers are designed to allow a $3.175 \mathrm{~mm}$-diameter microphone to be inserted between each individual divider plate. 


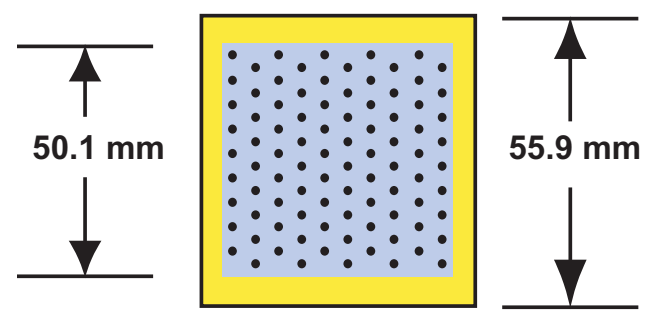

Figure 3. Sketch of porous honeycomb liner.

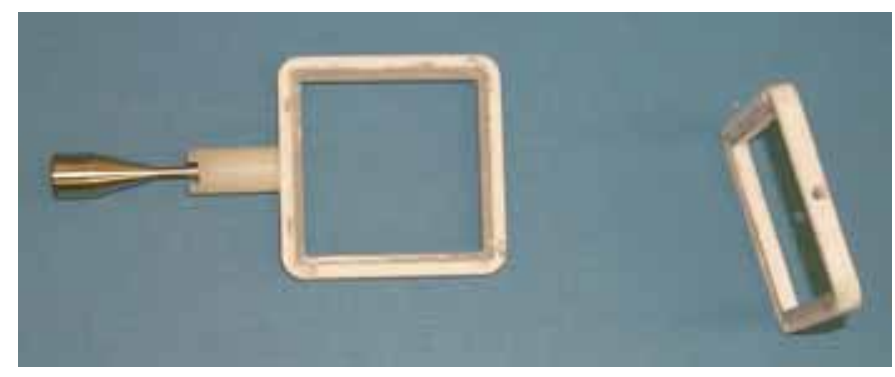

Figure 4. Photograph of spacers used to separate perforates (left spacer contains flush-mounted 3.175 mm-diameter microphone, right spacer shows port for microphone).

\section{Test Procedures}

\section{A. Two-Microphone Method (TMM)}

$\mathrm{T}$ HE Two-Microphone Method ${ }^{8,9}$ was used with the Langley normal incidence tube (see figure 5) to educe the impedance of each multi-layer perforate. These data were acquired with two acoustic source types: discrete frequency tones and random noise. For the discrete frequency tone source, data were acquired for one source frequency at a time, for source frequencies from 500 to $3000 \mathrm{~Hz}$, in increments of $100 \mathrm{~Hz}$. At each test frequency, reference sound pressure levels (SPL at the reference microphone; see figure 5) of 120 and $140 \mathrm{~dB}$ were tested. As stated earlier, since the thickness-to-hole diameter ratio was only 5 , there was some concern that the higher source amplitudes might cause nonlinear results to occur. This range of SPL's was chosen to allow an evaluation of the nonlinearity of the test specimens (multi-layer perforates), by determining whether the impedance is a function of the reference SPL. Similarly, for the random noise source, the overall sound pressure level (OASPL, integrated over frequency range of 500 to $3000 \mathrm{~Hz}$ ) was set to 100,120 and $140 \mathrm{~dB}$, for a sequence of three data sets. For this source, data were acquired at frequencies from 500 to $3000 \mathrm{~Hz}$, in increments of $12.5 \mathrm{~Hz}$. In all, there are five data sets (two for tone source, three for random noise source) for a single test specimen. Eight test specimens (multi-layer perforates, with 1, 3, 6, 12, 24, 36,48 and 64 porous plates) were tested at three porosities $(5,10$ and $15 \%)$ for a total of 120 test runs.

For the 64-layer sample consisting of perforates with a porosity of $10 \%$, one additional test was conducted using the Scott method. ${ }^{6}$ For this test procedure, measurements in the air space between adjacent perforates were used to locate a region where only progressive waves (into the sample) are dominant. Measures of the decay rates of the amplitude and phase of sound waves were then used to directly determine the propagation constant. For data where the amplitude and phase decay rates can be confidently determined, the propagation constant educed using the Scott Method can be used as a basis for evaluating the validity of the corresponding result educed using the Two-Thickness Method.

\section{B. Two-Thickness Method (TTM)}

$\mathrm{T}$

HE Two-Thickness Method ${ }^{5}$ (TTM) is well established for educing the bulk properties (characteristic impedance, $\xi_{c}$, and propagation constant, $\Gamma$ ) for homogeneous absorbing structures that can be regarded as a continuum from the standpoint of acoustic wave propagation. It has not been established that the TTM can be properly applied to a structures that cannot be reasonably regarded as a continuum, such as it the case for the multi-layer perforate samples of interest in this study. However, it is plausible to assume that the TTM would be expected to provide useful estimates 


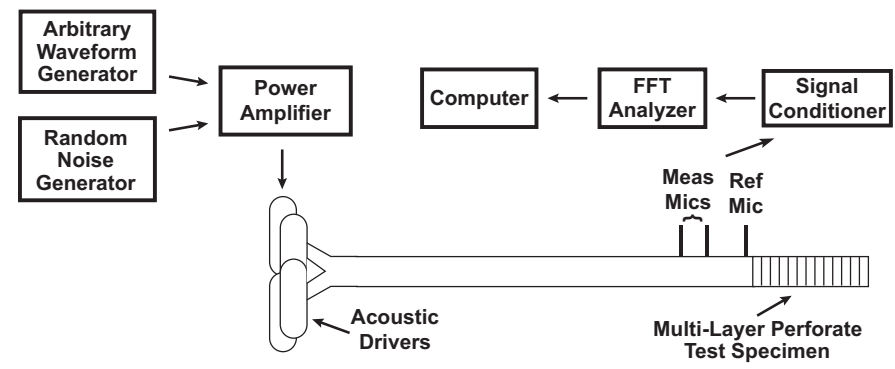

Figure 5. Sketch of Langley Normal Incidence Tube with Supporting Instrumentation.

of "smeared bulk properties" for structures where the wavelength-to-structure (structure scale taken to be the length of a layer, consisting of a single porous plate and an air space) scale ratio is sufficiently large. The primary goal of this study is to assess the capability of the TTM to provide these "smeared bulk properties" up to the measurement frequency limit of $3000 \mathrm{~Hz}$; i.e., for a wavelength-to-structure scale ratio of about 15. As a rough "rule-of-thumb," one might expect the continuum assumption to hold down to a ratio of about 10. Therefore, it is reasonable to expect that TTM to provide "good estimates" of the smeared bulk properties. It is critical that a convincing validation scheme be devised to provide a level of confidence in the results. The L2-Norm, commonly used in the numerical analysis community, is used in this study for this purpose.

The core of the TTM is the solution of the following two equations:

$$
\begin{aligned}
& \zeta_{1}=\zeta_{c} \operatorname{coth}\left(\Gamma d_{1}\right) \\
& \zeta_{2}=\zeta_{c} \operatorname{coth}\left(\Gamma d_{2}\right)
\end{aligned}
$$

where $\zeta_{1}$ and $\zeta_{2}$ represent two measured surface impedances on two separate test samples of depths $d_{1}$ and $d_{2}$, taken from what is assumed to be a homogeneous, continuous structure. In the current study, the analysis is greatly simplified by selecting sample depths such that $d_{2}=2 d_{1}$. Specifically, four pairs of samples ( 3 and 6 layers, 6 and 12 layers, 12 and 24 layers, and 24 and 48 layers) were used as input for the TTM.

Given the two measured impedance spectra, $\zeta_{1}$ and $\zeta_{2}$, acquired at two depths such that $d_{2}=2 d_{1}$, the characteristic impedance and propagation constant can be determined (as a function of frequency) as follows:

$$
\begin{gathered}
\zeta_{c}=\sqrt{\zeta_{1}\left(2 \zeta_{2}-\zeta_{1}\right)} \\
\Gamma=\Gamma_{r}+I \Gamma_{i}=\frac{1}{2 d_{1}} \log \frac{1+a}{1-a}
\end{gathered}
$$

where

$$
a=\sqrt{\frac{2 \zeta_{2}-\zeta_{1}}{\zeta_{1}}}
$$

From these two complex quantities, the attenuation rate and phase rate can be determined. The attenuation rate $\left(8.686 \Gamma_{r}\right.$, in $\left.\mathrm{dB} / \mathrm{m}\right)$ is the rate at which the amplitude of the acoustic wave decays as is travels through the sample. Correspondingly, the phase rate $\left(\Gamma_{i}\right.$, in $\left.\mathrm{rad} / \mathrm{m}\right)$ is the rate at which the phase of the acoustic wave varies as it travels through the sample. If the parent material of the two initial samples (in this case, multiple layers of perforated plates) is sufficiently uniform, such that the intrinsic properties of the material can be successfully educed from these two samples, the impedance $\zeta_{s}$ of a third sample with thickness $d_{s}$ can then be determined using

$$
\zeta_{s}=\zeta_{c} \operatorname{coth}\left(\Gamma d_{s}\right)
$$

A comparison between the measured impedance of the third sample and the impedance predicted using the $\zeta_{c}$ and $\Gamma$ educed using the TTM can be used to assess the "validity" of the educed parameters. The L2-Norm, $L 2$, is used for this evaluation. It is computed as follows: 


$$
L 2=\frac{1}{M} \sqrt{\sum_{i}^{M}\left|\zeta_{m, i}-\zeta_{p, i}\right|^{2}}
$$

where $\zeta_{m, i}$ and $\zeta_{p, i}$ are the measured and predicted impedances, respectively, at the $i^{\text {th }}$ frequency, and $M$ is the total number of frequencies used in the evaluation. Ideally, the value of $L 2$ should be zero, indicating exact comparison between the measured and predicted impedances for samples with depths that are different from those used as input to the method. (By definition, the value of $L 2$ should be zero for comparisons based on one of the two sample thickness used as input to the L2-Norm computation.) Thus, any departure from zero is a measure of (1) experimental error, and (2) a breakdown of the continuum assumption (e.g., material imperfections). For the current study, no attempt is made to differentiate between these sources of error. It is hoped that $L 2$ is sufficiently small that there is no need to sort out the causes of error. Realistically, if the value of $L 2$ is no greater than 0.1 , which indicates the average error between the measured and predicted impedances is no greater than approximately 0.1 (impedances normalized by $\rho c$ ), the multi-layer perforates used in this study will be considered to be properly described as "bulk absorbers."

\section{Results and Discussion}

\section{A. Normalized Impedance}

A $\mathrm{S}$ indicated earlier, tests were conducted with samples consisting of perforated plates with three porosities $(5,10$ A and 15\%). These samples were tested with two source types, a discrete frequency tone source and a random noise source. Similar results were observed for both source types. Thus, for the sake of brevity, random noise data will be used for the majority of the remainder of the paper, with the sole exception of the implementation of the Scott Method results. This method is much more amenable to the usage of discrete tone data. Figures 6 and 7 (for convenience, these and all successive figures are provided at the end of this paper) provide the normalized acoustic resistance, $\theta$, and reactance, $\chi$, spectra for multi-layer samples (a layer consists of one porous plate and one air space, with a combined thickness of $6.731 \mathrm{~mm}$ ) with $5 \%$ porous plates, acquired with a random noise source with an OASPL of $120 \mathrm{~dB}$. Similarly, figures 8 through 11 provide normalized impedance $\left(\zeta_{s}=\theta+I \chi\right)$ results acquired with the same source, with 10 and $15 \%$ porous plates. For this constant source amplitude, the reduction in mass reactance that occurs as the porosity of the plates is increased from 5 to $15 \%$ results in a corresponding increase in resonance frequency. As expected, the resistance is also observed to decrease with increasing porosity.

These impedance data also demonstrate a consistent trend, regardless of the porosity of the plates used to form the multi-layer samples. As the number of layers is increased for any of the three porosities tested, two patterns emerge. For discussion, refer to the $10 \%$ porous plate data in figures 8 and 9. Note that the lowest resonance (first positivegoing zero crossing in reactance spectra) decreases as the number of layers is increased from 3 to 12. Correspondingly, the anti-resonance behavior becomes subdued. For the resistance, the peaks in the vicinity of anti-resonance become less pronounced and the resistance increases to "fill in the valleys." Similar trends occur in the reactance spectra. For greater than 12 layers, the latter of these effects (flattening of peaks) appears to become the dominant feature, thus inhibiting the reactance from reaching zero for multiple resonances. This combination of effects is apparent for each of the porosities tested.

Figures 12 and 13 provide the acoustic impedance spectra acquired with 10\% porous plates with an OASPL of $100 \mathrm{~dB}$, and figures 14 and 15 provide the corresponding results acquired at an OASPL of $140 \mathrm{~dB}$. When compared with figures 8 and 9, these results provide a measure of the effects of source amplitude on the acoustic impedance for the same samples. In general, the results are shown to be very similar, indicating the $10 \%$ porosity samples are very linear.

\section{B. L2-Norm for $\left|\zeta_{m}-\zeta_{p}\right|$}

$\mathrm{T}$ HE measured impedances of these multi-layer perforate samples were used as input to the TTM to determine intrinsic acoustic characteristics. As stated earlier, if the test samples are constructed with uniform material that can properly be described via intrinsic properties (e.g., characteristic impedance and propagation constant), the impedance of samples constructed with different depths of the same material should be easily predicted. As shown in figures 16 through 21, it is important to properly choose the thickness of the two samples used as input for the TTM. In these figures, the L2-Norm, $L 2$, of the difference between the measured and predicted impedances is provided. Figure 16 provides the L2-Norm as a function of the number of layers used in the test sample. The different colored bars represent the results determined using the TTM for various pairs of samples. 
For example, the blue bars in figure 16 provide the results educed using the 3-layer $(d=20.2 \mathrm{~mm})$ and 6-layer $(2 d=40.4 \mathrm{~mm})$ samples (indicated as $(3,6)$ in the figure). These two samples were used as input to the TTM, which was used to compute the characteristic impedance, $\zeta_{c}$, and propagation constant, $\Gamma$. These parameters were then used to "predict" the impedance for selected thicknesses $(3,6,12,24,36,48$ and 64 layers; corresponding to thicknesses of $20.2,40.4,80.8,161.5,242.3,323.1$ and $430.8 \mathrm{~mm}$ ). Since the 3 and 6-layer liners were the two samples used as input to the TTM, the L2-Norms are identically zero for those liners. For the 12-layer liner, the L2-Norm is 0.028, which indicates that the average difference between the predicted and measured values of $\zeta$ is expected to be approximately 0.028 . The L2-Norms for liners consisting of a larger $(>12)$ number of layers are even lower $(0.021$ or less). These values of $L 2$ are significantly less than the value of 0.1 used as the criteria for determining if the TTM is providing quality results. Clearly, these excellent comparisons indicate the $(3,6)$ pair is a good choice for input to the TTM.

The red bars in figure 16 show the results of educing the characteristic impedance and propagation constant spectra from measured impedances for the 6 and 12-layer samples (indicated as $(6,12)$ in the figure and in the remainder of this paper). As required, the values are zero for 6 and 12-layer samples. Again, this indicates the process is properly predicting the impedances of the two samples used as input to the TTM. When the $\zeta_{c}$ and $\Gamma$ spectra are used to predict the impedance of the 3-layer sample, the L2-Norm indicates an error of 0.027. This is very similar to the result achieved using the $(3,6)$ pair to predict the impedance of the 12-layer liner. However, the errors in predicting the larger number $(\geq 24)$ of layers with the $(6,12)$ pair are noticeably smaller than those based on the $(3,6)$ pair. The yellow bars provide the results based on the $(12,24)$ pair $(12$ and 24-layer liners). They follow similar trends to those achieved with the $(3,6)$ and $(6,12)$ pairs. For the $(24,48)$ pair, however, the differences between the predicted and measured impedances are clearly much higher. When the $(24,48)$ pair is used as input for the TTM, the resultant $\zeta_{c}$ and $\Gamma$ spectra do a poor job of predicting the impedances of the 3, 6 and 12-layer liners. The L2-Norm for the 3-layer liner is 0.244 , which greatly exceeds the scale of the figure (scale chosen to allow comparison of the details for the best datasets). This is not entirely unexpected. As the samples used as input to the TTM become increasingly thick, the impedances of the two samples converge. Looking back at figures 6 and 7, this is clearly the case for this porosity and source amplitude. The use of two nearly identical impedances as input to the TTM results in a set of ill-conditioned equations, from which poor results should be expected. Thus, the $(24,48)$ pair should not be used for this combination of porosity (all of the plates are 5\% porous) and source amplitude (120 dB OASPL).

Figure 17 contains similar results for samples consisting of $15 \%$ porous plates, with the source amplitude maintained at an OASPL of $120 \mathrm{~dB}$. Looking back at figures 10 and 11, the impedances for the various pairs of sample do not converge. For example, the impedances of the 24 and 48-layer liners (depicted as blue and red in figures 10 and 11) can be easily distinguished. As a result, the L2-Norm results shown in figure 17 are all within acceptable limits. Based on these results, the $(6,12)$ and $(12,24)$ pairs provide the best input for the TTM. Figures 18 and 19 provide similar results for samples consisting of $10 \%$ porous plates, with the source amplitude increasing from $100 \mathrm{~dB}$ OASPL to $140 \mathrm{~dB}$ OASPL. For these configurations, the choice of sample pairs to use as input to the TTM is not critical, as all of the results indicate L2-Norms of less than 0.024 .

The results provided above were for (1) constant source amplitude and varying porosity (figures 16 and 17) and (2) constant porosity and varying source amplitude (figures 18 and 19). In the following, the two limiting conditions are explored. For the first, the porosity is set to the minimum value (5\%) and the source amplitude is set to the maximum value (140 dB OASPL). This represents the most nonlinear condition achievable with the current dataset. For comparison, the second configuration consists of plates with maximum porosity (15\%) and minimum source amplitude (100 dB OASPL). This is expected to be the most linear condition included in the current dataset. Figure 20 provides the L2-Norm results for the nonlinear configuration (5\% porosity, $140 \mathrm{~dB}$ OASPL). The $(24,48)$ pair is again shown to be a poor basis for the TTM, based on the poor comparisons for the 3, 6 and 12-layer samples. While all of the other pairs provide acceptable results, it is the opinion of the authors that the $(6,12)$ pair is preferred. From a practical point of view, it is ideal to be able to use the minimum number of plates as input to the TTM (less cost to fabricate, easier to assemble). The results provided in figure 21 for the most linear configuration are not as dependent on the pair of samples chosen as input to the TTM. However, the choice of the $(6,12)$ pair appears to also be appropriate for this condition.

Clearly, the excellent comparisons between measured and predicted impedances for the majority of the cases included in this study demonstrate that, at least for this set of perforates, multi-layer perforates can be acceptably treated as bulk absorbers.

\section{Educed Intrinsic Parameters}

FIGURES 22 through 27 provide the intrinsic parameters determined with the Two-Thickness Method for each pair of samples, for the nonlinear (5\% porosity, $140 \mathrm{~dB}$ OASPL) and linear (15\% porosity, $100 \mathrm{~dB}$ OASPL) configurations, 
respectively. Figures 22 and 23 provide the magnitude of the characteristic impedance as a function of frequency. For comparison, the corresponding value for air is unity. For the nonlinear condition, the magnitude of $\zeta_{c}$ hovers around 2.5 to 3.0, whereas it drops to approximately 1.7 for the linear condition. As discussed earlier, the propagation constant, $\Gamma$, can be used to determine the attenuation rate within the sample. Figure 24 shows the attenuation rate to increase from approximately $100 \mathrm{~dB} / \mathrm{m}$ at $500 \mathrm{~Hz}$ to approximately $165 \mathrm{~dB} / \mathrm{m}$ at $3000 \mathrm{~Hz}$. (Recall that the $(24,48$ ) pair results in ill-conditioned equations and should not be used.) Similarly, figure 25 indicates the attenuation rate for the linear configuration to range from approximately $60 \mathrm{~dB} / \mathrm{m}$ at $500 \mathrm{~Hz}$ to approximately $70 \mathrm{~dB} / \mathrm{m}$ at $3000 \mathrm{~Hz}$. Finally, figures 26 and 27 provide the ratio of sound speed through the material, $c_{m}$, to that in air, $c$. For the nonlinear configuration, the sound speed through the material is observed to hover between $40 \%$ and $50 \%$ the sound speed of air. For the linear configuration, the sound speed through the material increases to nearly $70 \%$ the sound speed of air.

The Scott method ${ }^{6}$ was used as an independent means of verifying the propagation constant results achieved via the TTM. This method requires measurement of the acoustic pressure at various depths in the material, such that the attenuation and phase decay rates can be determined directly. Figure 28 provides SPL and Phase distributions from measurements within a 64-layer sample consisting of $10 \%$ porous plates. These data were acquired with a $120 \mathrm{~dB}$ (measured at the reference microphone, see figure 5) discrete tone source, at frequencies of 500 and $3000 \mathrm{~Hz}$. Both decay rates are observed to be linear; thus, the Scott method should be suitable for this data. The attenuation rates at 500 and $3000 \mathrm{~Hz}$, as measured via the Scott method, are 71.1 and $101.4 \mathrm{~dB} / \mathrm{m}$, respectively. The corresponding attenuation rates educed via the TTM are 69.5 and $115.4 \mathrm{~dB} / \mathrm{m}$. The sound speed ratio (ratio of sound speed in material to that in air) educed using the Scott method ranges from 0.56 to 0.64 at 500 and $3000 \mathrm{~Hz}$, respectively. The corresponding ratios educed using the TTM were 0.55 and 0.62 . These results are well within the range of variability that is observed for the TTM results when different pairs of samples are used as input to the TTM.

For comparison, the Delany-Bazely semi-empirical model ${ }^{7,10}$ was used to determine the characteristic impedance and propagation constant for fiberglas fabricated by Owens-Corning Fiberglas Corporation. A comparison of $\zeta_{c}$ and $\Gamma$ for fiberglas with $5.1 \mu \mathrm{m}$-diameter fibers and a bulk density of $16 \mathrm{~kg} / \mathrm{m}^{3}$, with the corresponding values for a multilayer perforate consisting of $10 \%$ porous plates, is provided for two source frequencies (discrete tone source) in the table below:

\begin{tabular}{|c|c|c|c|c|c|c|c|c|}
\hline & \multicolumn{4}{|c|}{ Fiberglas } & \multicolumn{3}{c|}{ 10\% Multi-Layer Perforate } \\
\hline Freq, Hz & \multicolumn{2}{|c|}{$\xi_{c}$} & \multicolumn{2}{c|}{$\Gamma$} & \multicolumn{2}{c|}{$\xi_{c}$} & \multicolumn{2}{c|}{$\Gamma$} \\
\hline & Real & Imag & Real & Imag & Real & Imag & Real & Imag \\
\hline 500 & 1.40 & -0.57 & 7.97 & 14.56 & 2.11 & -0.99 & 8.69 & 16.33 \\
\hline 3000 & 1.10 & -0.15 & 16.46 & 64.20 & 2.28 & 0.17 & 13.35 & 90.72 \\
\hline
\end{tabular}

These data indicate the properties of the $10 \%$ porosity multi-layer perforate are similar to those of fiberglas with $5.1 \mu \mathrm{m}$-diameter fibers and a bulk density of $16 \mathrm{~kg} / \mathrm{m}^{3}$. This favorable comparison provides further confidence that the current set of multi-layer perforate liners can be acceptably treated as "bulk absorbers."

\section{Concluding Remarks}

N extensive database has been acquired using the NASA Langley Research Center Normal Incidence Tube to A evaluate a set of multi-layer perforates. These data were used as input to a Two-Thickness Method (TTM) analysis designed to educe intrinsic acoustic parameters (characteristic impedance and propagation constant) for the multi-layer perforates. This method assumes the test samples to consist of bulk materials. Therefore, if these intrinsic parameters can be used to correctly predict the normal incidence impedance of other thicknesses (in this case, a different number of perforate layers), the samples can be treated as "bulk" materials.

For the current study, the multi-layer perforates consisted of porous plates with porosities of 5,10 and $15 \%$, separated by air gaps such that they were $6.731 \mathrm{~mm}$ apart. Samples consisting of 1, 3, 6, 12, 24, 36, 48 and 64 porous plates were tested, with discrete tone and random noise sources. Pairs of samples ( 3 and 6, 6 and 12, 12 and 24, 24 and 48 layers) were used with the TTM to determine the characteristic impedance and propagation constant spectra for multi-layer perforates. These quantities were then used to predict the normal incidence acoustic impedance of different multi-layer perforates. The following results were observed:

1. For a constant source amplitude, the resonance frequency increases and the acoustic resistance decreases as the porosity of the constituent plates is increased from 5 to $15 \%$. 
2. For samples consisting of $10 \%$ porous plates, minimal variability in the acoustic impedance is observed as the source amplitude is increased from 100 to $140 \mathrm{~dB}$ OASPL. This indicates these samples are very linear.

3. For the range of porosity used in this study, the 6 and 12-layer pair of samples provides the best input for TTM. This is evidenced by the excellent comparison between "predicted" (based on intrinsic parameters educed using TTM) and measured impedances for the samples with a different number of layers. Good results were also achieved with the 12 and 24-layer pair; however, due to mechanical complexity, the $(6,12)$ pair is preferred.

4. As the test condition becomes more nonlinear, due to a reduction in porosity and/or an increase in source amplitude, the $(24,48)$ pair of samples fails to provide suitable input for the TTM. This results from the fact that the normal incidence impedance spectra for these two thicknesses are nearly identical, causing the equations used in the TTM to be ill-conditioned.

5. As the porosity is decreased from 15 to $5 \%$ and the source amplitude is increased from 100 to $140 \mathrm{~dB}$ OASPL, (1) the magnitude of the characteristic impedance increases from approximately 1.7 to 3.0 , (2) the average attenuation rate increases from 65 to $130 \mathrm{~dB} / \mathrm{m}$, and (3) the sound speed through the material (multiple layers of porous plates separated by air gaps) decreases from 70 to $45 \%$ of the sound speed in air.

6. The Scott method can be used to educe results similar to those achieved with the Two-Thickness Method. This provides increased confidence in the Two-Thickness Method results.

7. Based on results calculated using the Delany-Bazely method, the properties of the $10 \%$ porosity multi-layer perforate are similar to those of fiberglas with $5.1 \mu \mathrm{m}$-diameter fibers and a bulk density of $16 \mathrm{~kg} / \mathrm{m}^{3}$.

The excellent comparisons between measured and predicted impedances for the majority of the cases included in this study demonstrate that, at least for this set of perforates, multi-layer perforates can be acceptably treated as bulk absorbers. The authors plan to use this data to validate an analytical model being developed at Langley (presented in a companion paper), with the intention of using the resultant model to design optimized liners for broadband noise reduction.

\section{Acknowledgments}

$T^{H E}$ authors would like to express their appreciation to Carol Harrison and Brian Howerton for their support in the acquisition of the dataset used in this investigation.

\section{References}

${ }^{1}$ Lester, H.C., Preisser, J.S., and Parrott, T.L., "Design and Flight Test of a Kevlar Acoustic Liner," Journal of Aircraft, Vol. 21, No. 7, 1984, pp. 491-497.

${ }^{2}$ Bielak, G.W., Premo, J.W., and Hersh, A.S., “Advanced Turbofan Duct Liner Concepts," NASA CR-1999-209002, 1999. 2004

${ }^{3}$ Hillereau, N., "Suppression Characteristics of Acoustic Liners with Porous Honeycomb," Master's Thesis from University of Cincinnati,

${ }^{4}$ Parrott, T.L. and Jones, M.G., "Cascaded Perforates as One-Dimensional, Bulk Absorbers," AIAA-2006-2402, presented at 12 th Aeroacoustics Conference, Cambridge, MA, May, 2006.

${ }^{5}$ Smith, C.D. and Parrott, T.L., "Comparison Of Three Methods For Measuring Acoustic Properties Of Bulk Materials," Journal of Acoustical Society of America, Vol. 74, No. 5, 1983, pp. 1577-1582.

${ }^{6}$ Scott, R.A., “The Absorption of Sound in a Homogeneous Porous Medium,” Proceedings of the Physical Society, Vol. 58, 1946, pp. 165-183.

${ }^{7}$ Delany, M.E. and Bazley, E.N., "Acoustical Properties of Fibrous Absorbent Materials," Applied Acoustics, Vol. 3, 1970, pp. $105-116$.

${ }^{8}$ Chung, J.Y. and Blaser, D.A., "Transfer function method of measuring in-duct acoustic properties: I. Theory," Journal of Acoustical Society of America, Vol. 68, 1980, pp. 907-921.

${ }^{9}$ Jones, M.G. and Parrott, T.L., "Evaluation of a multi-point method for determining acoustic impedance," Journal of Mechanical Systems and Signal Processing, Vol. 3, No. 1, 1989, pp. 15-35.

${ }^{10}$ Beranek, L.L., Noise and Vibration Control, McGraw-Hill Book Company, 1971. 


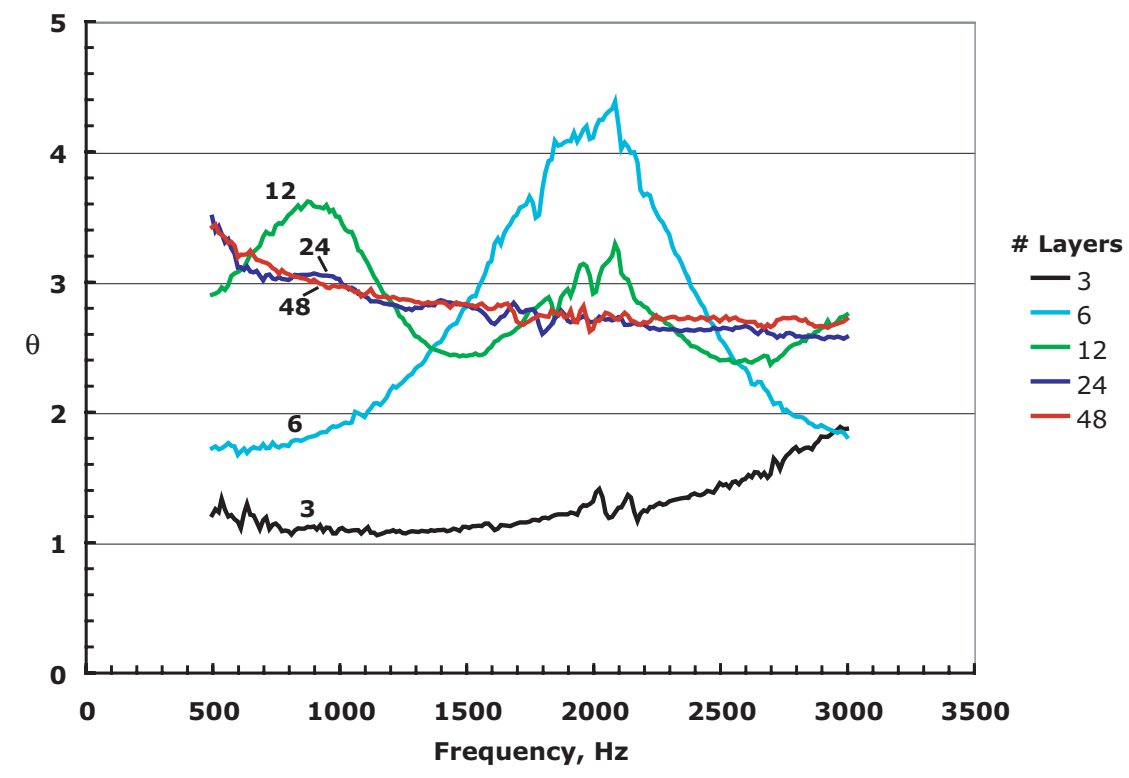

Figure 6. Normal incidence resistance spectra (normalized by $\rho c$ ) of multi-layer liners consisting of $5 \%$ porous perforated plates (reference OASPL of $120 \mathrm{~dB}$ ).

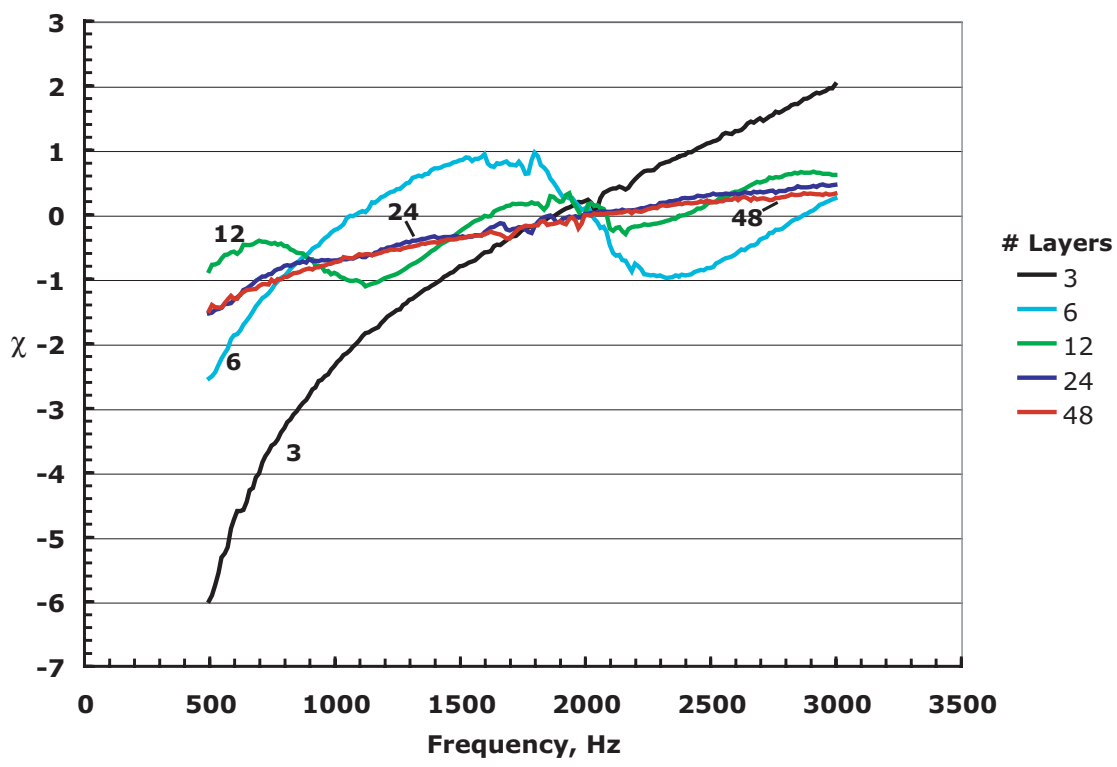

Figure 7. Normal incidence reactance spectra (normalized by $\rho c$ ) of multi-layer perforate liners consisting of $5 \%$ porous perforated plates (reference OASPL of $120 \mathrm{~dB}$ ). 


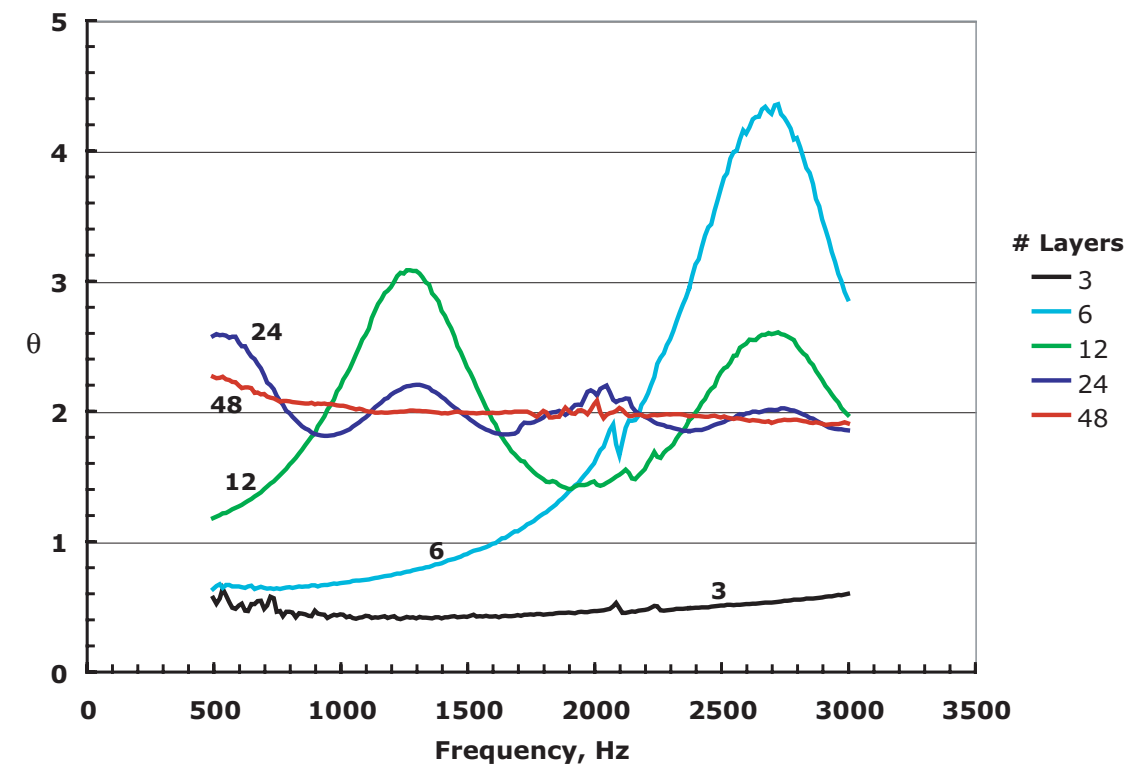

Figure 8. Normal incidence resistance spectra (normalized by $\rho c$ ) of multi-layer liners consisting of $10 \%$ porous perforated plates (reference OASPL of $120 \mathrm{~dB})$.

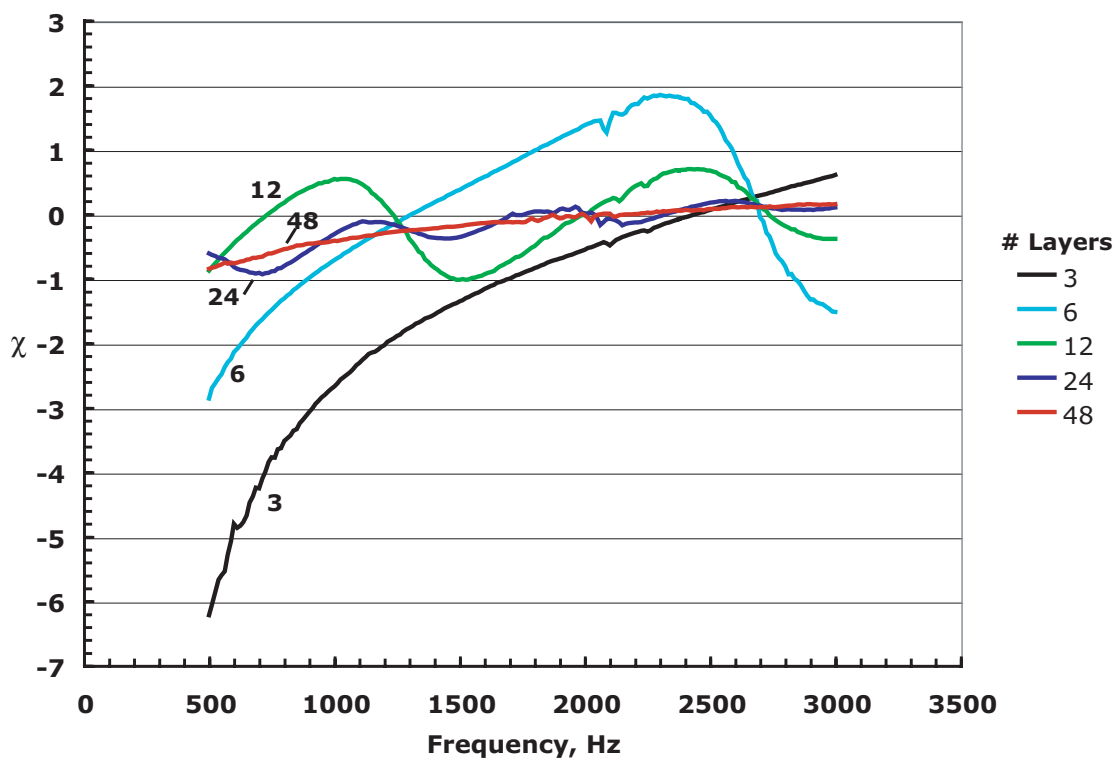

Figure 9. Normal incidence reactance spectra (normalized by $\rho c$ ) of multi-layer perforate liners consisting of $10 \%$ porous perforated plates (reference OASPL of $120 \mathrm{~dB}$ ). 


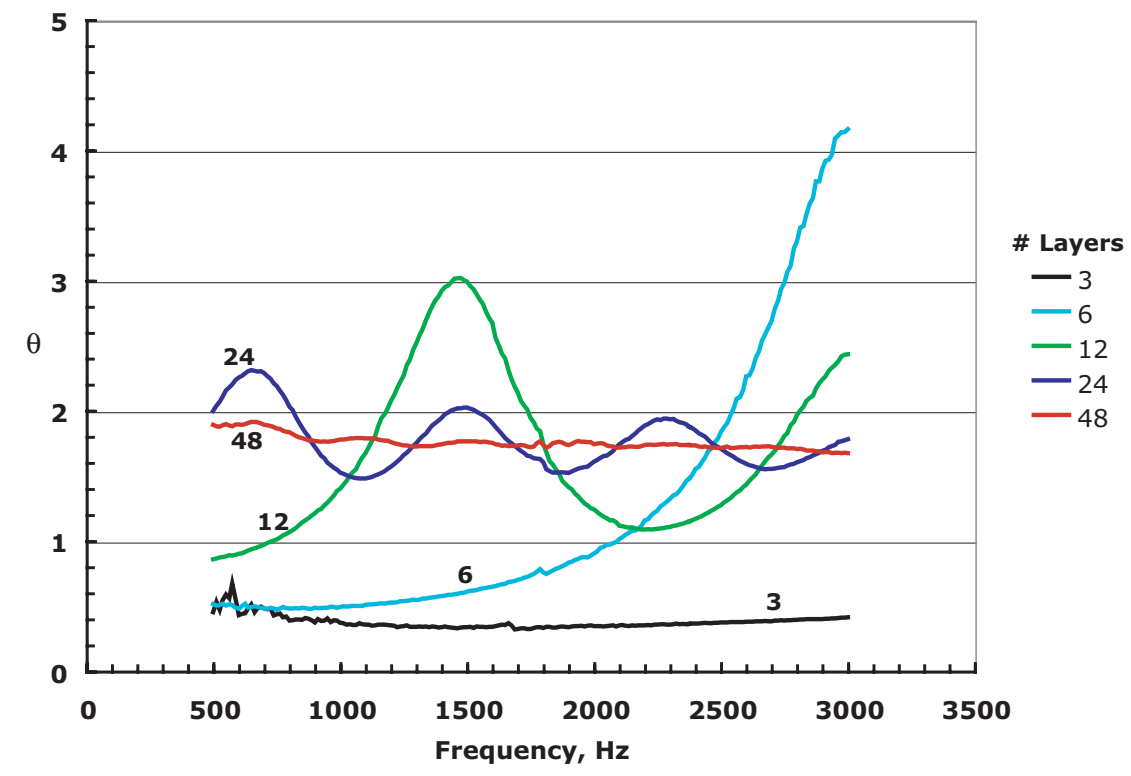

Figure 10. Normal incidence resistance spectra (normalized by $\rho c$ ) of multi-layer liners consisting of $15 \%$ porous perforated plates (reference OASPL of $120 \mathrm{~dB}$ ).

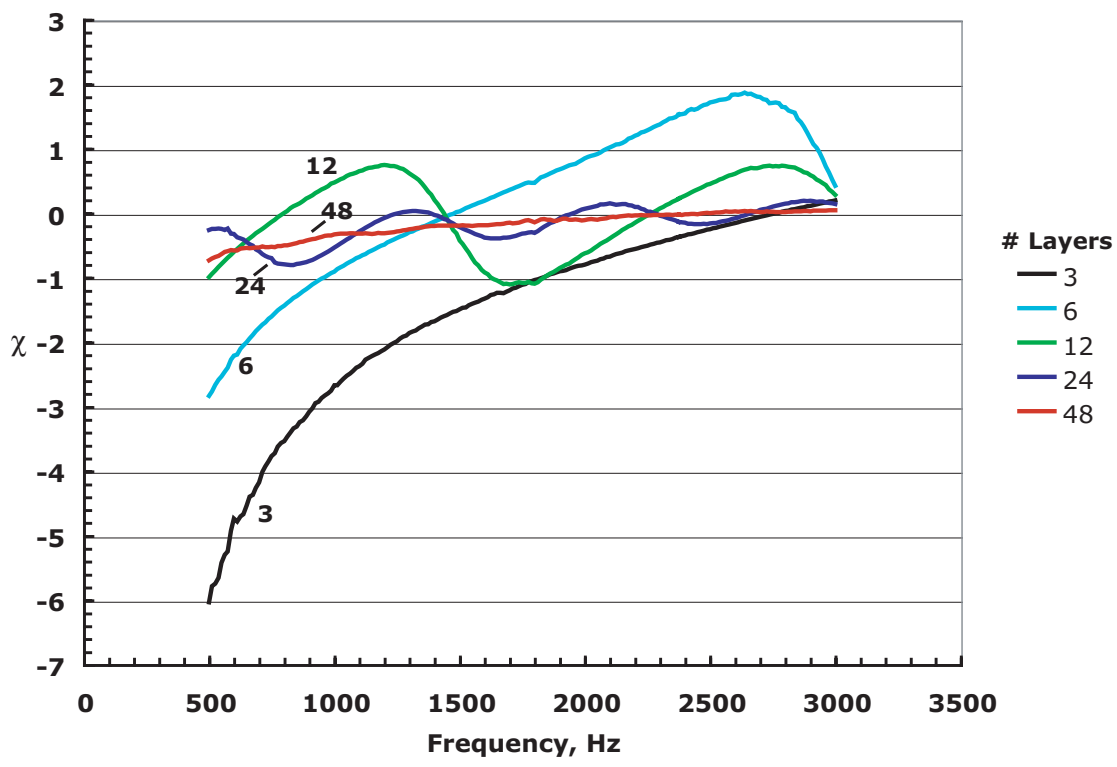

Figure 11. Normal incidence reactance spectra (normalized by $\rho c$ ) of multi-layer perforate liners consisting of $15 \%$ porous perforated plates (reference OASPL of $120 \mathrm{~dB}$ ). 


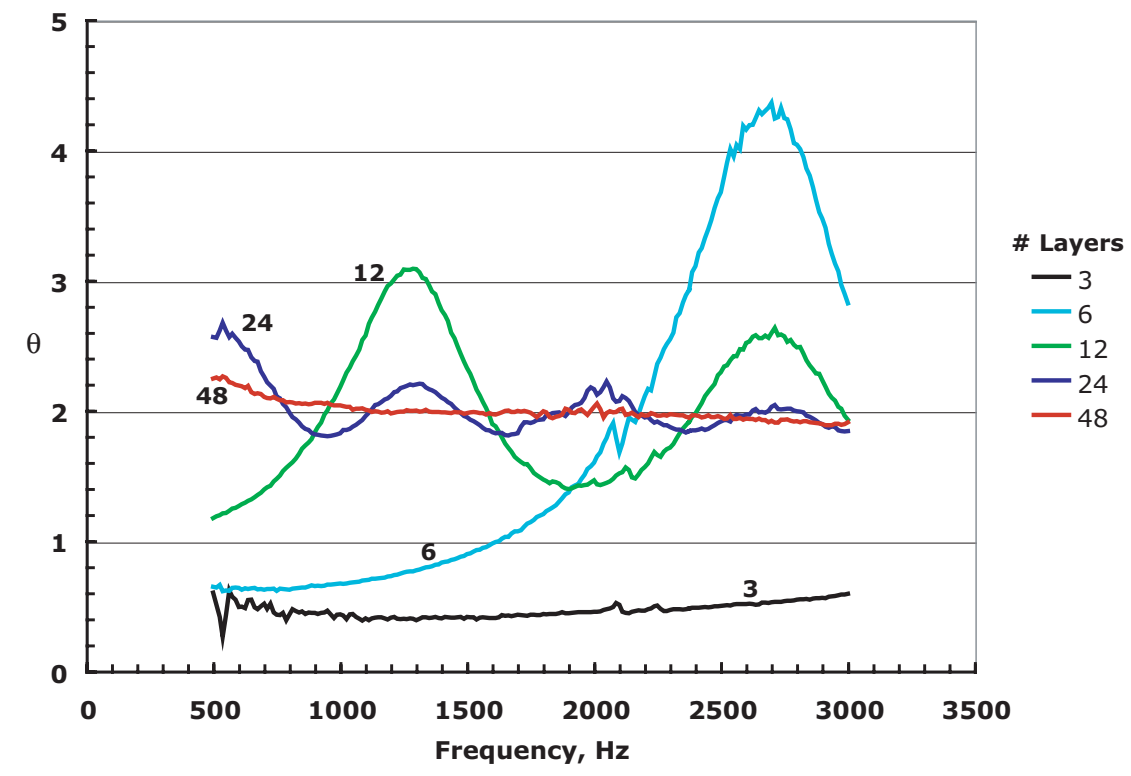

Figure 12. Normal incidence resistance spectra (normalized by $\rho c$ ) of multi-layer liners consisting of $10 \%$ porous perforated plates (reference OASPL of $100 \mathrm{~dB}$ ).

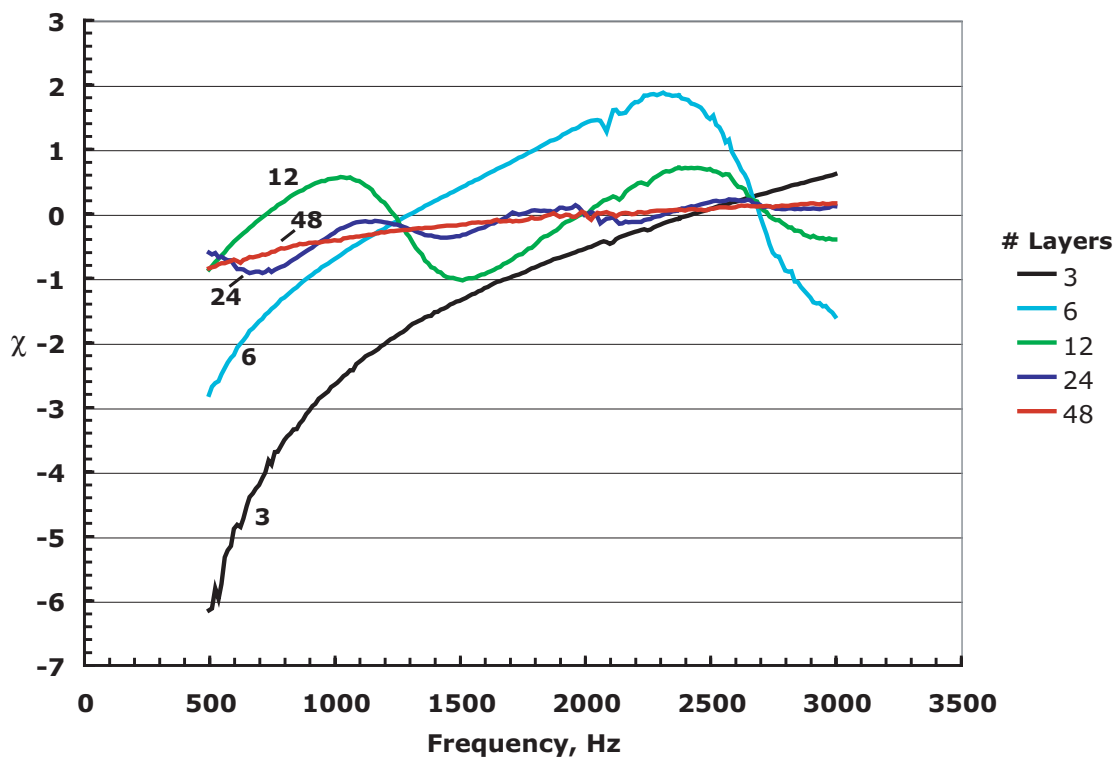

Figure 13. Normal incidence reactance spectra (normalized by $\rho c$ ) of multi-layer perforate liners consisting of $10 \%$ porous perforated plates (reference OASPL of $100 \mathrm{~dB}$ ). 


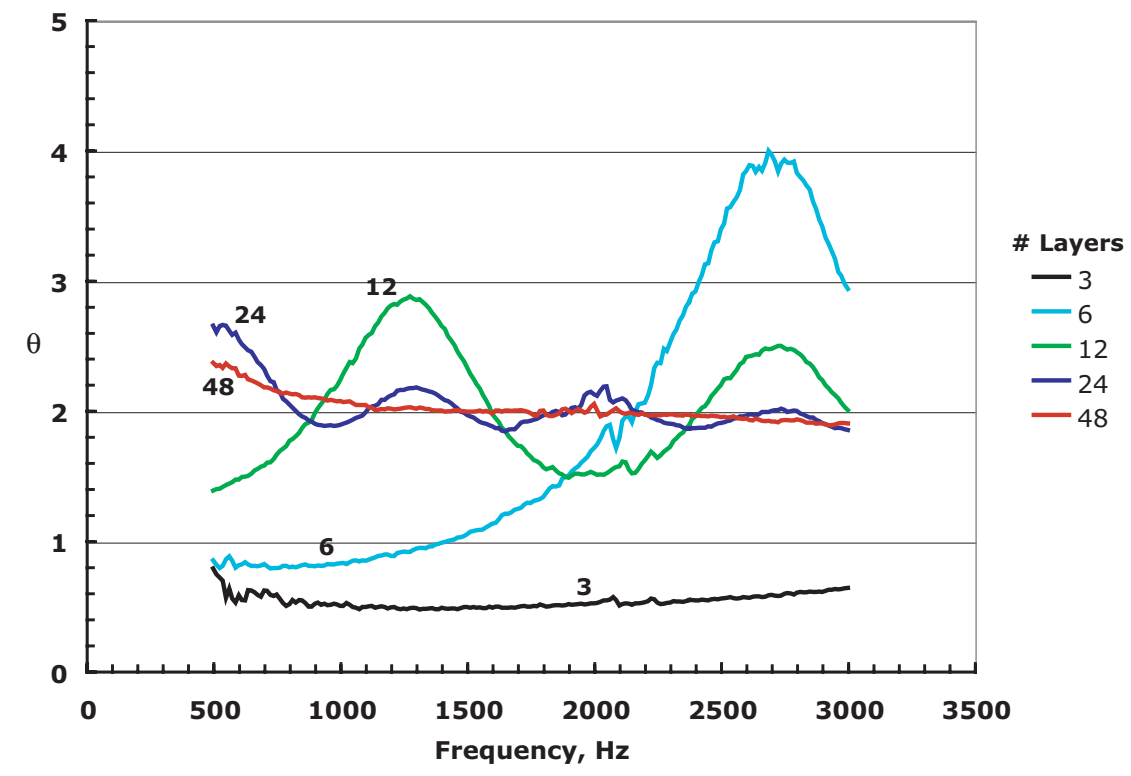

Figure 14. Normal incidence resistance spectra (normalized by $\rho c$ ) of multi-layer liners consisting of $10 \%$ porous perforated plates (reference OASPL of $140 \mathrm{~dB})$.

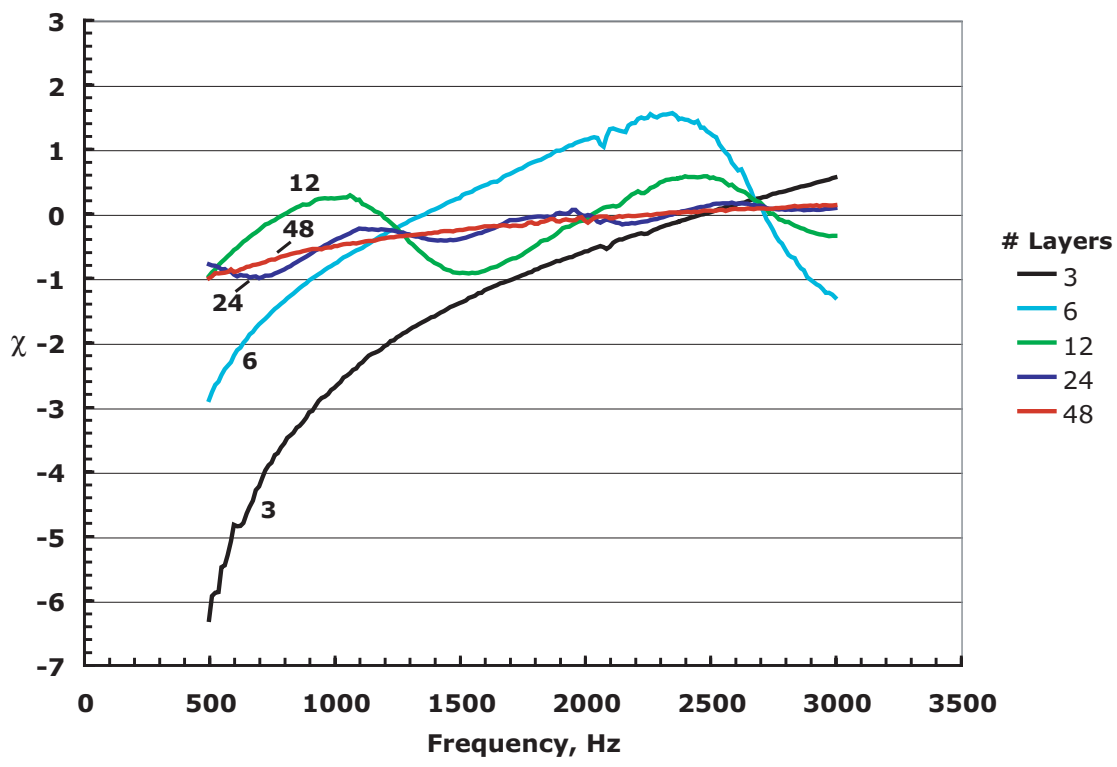

Figure 15. Normal incidence reactance spectra (normalized by $\rho c$ ) of multi-layer perforate liners consisting of $10 \%$ porous perforated plates (reference OASPL of $140 \mathrm{~dB}$ ). 


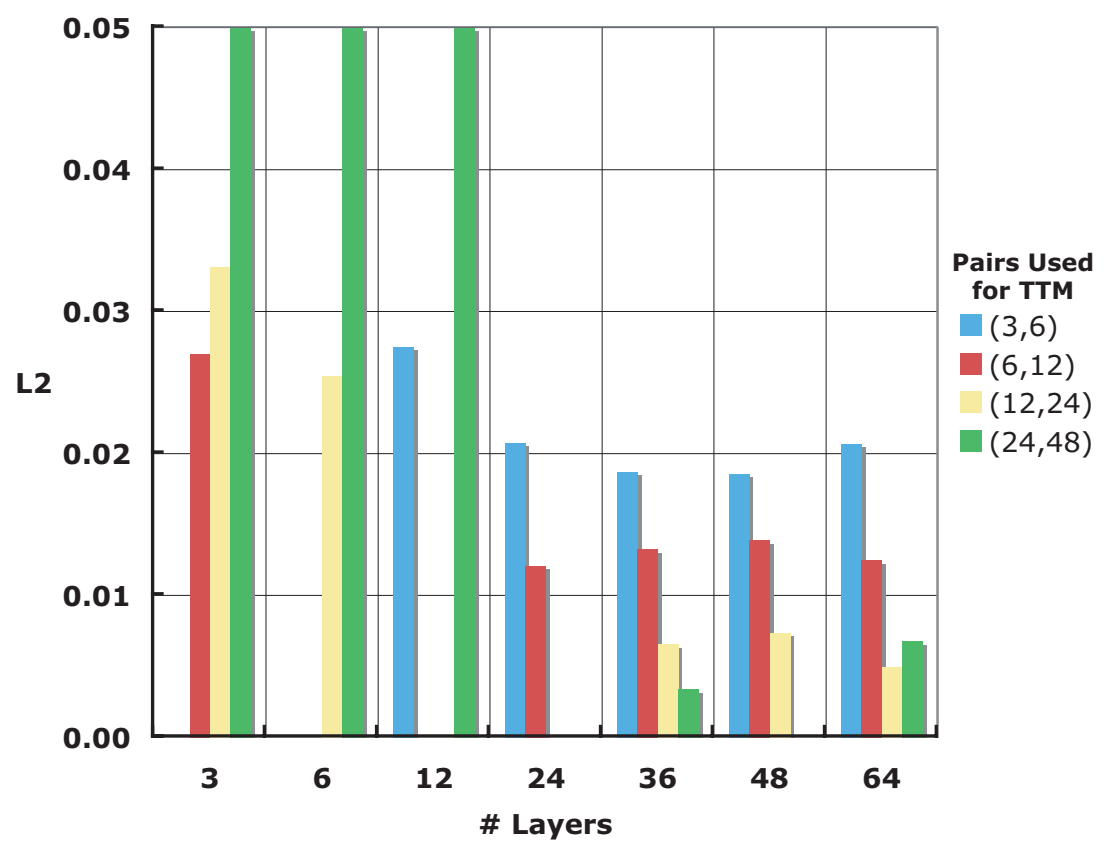

Figure 16. L2-Norm for $\left|\zeta_{m}-\zeta_{p}\right|$, for multi-layer perforate liners consisting of $5 \%$ porous perforated plates (reference OASPL of $120 \mathrm{~dB}$ ).

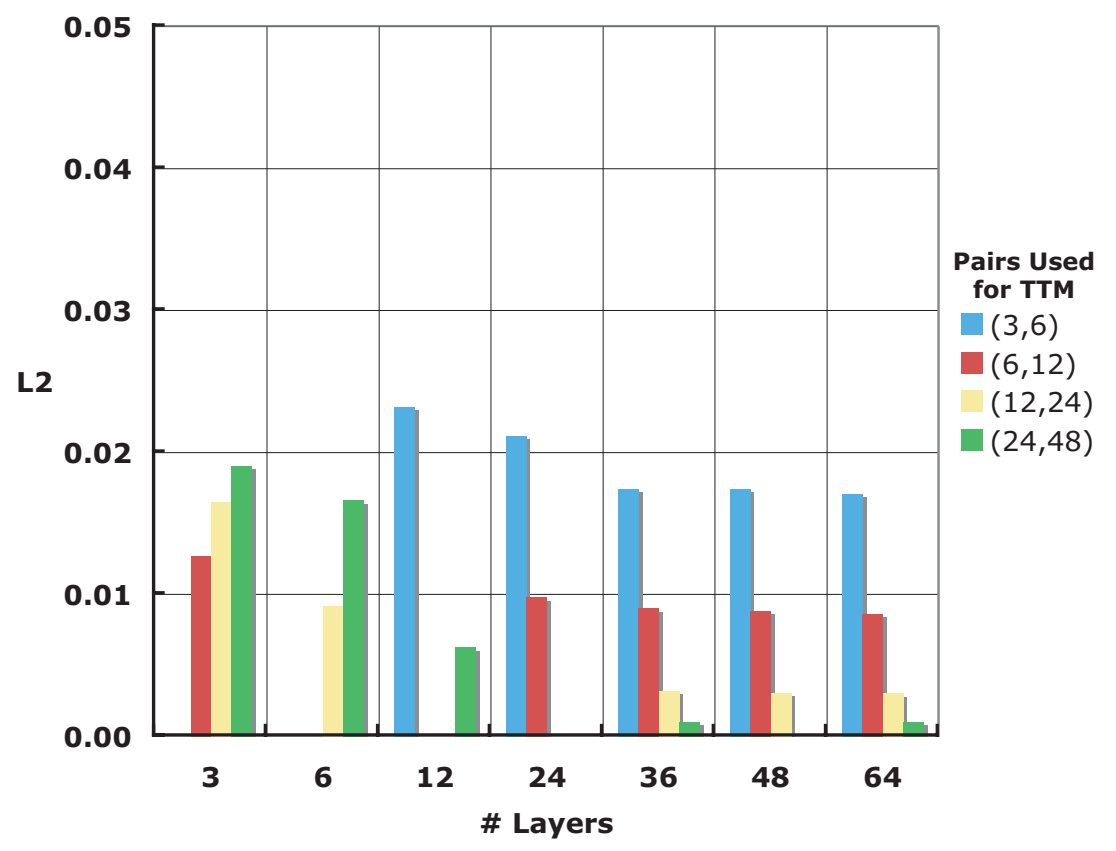

Figure 17. L2-Norm for $\left|\zeta_{m}-\zeta_{p}\right|$, for multi-layer perforate liners consisting of 15\% porous perforated plates (reference OASPL of $120 \mathrm{~dB}$ ). 


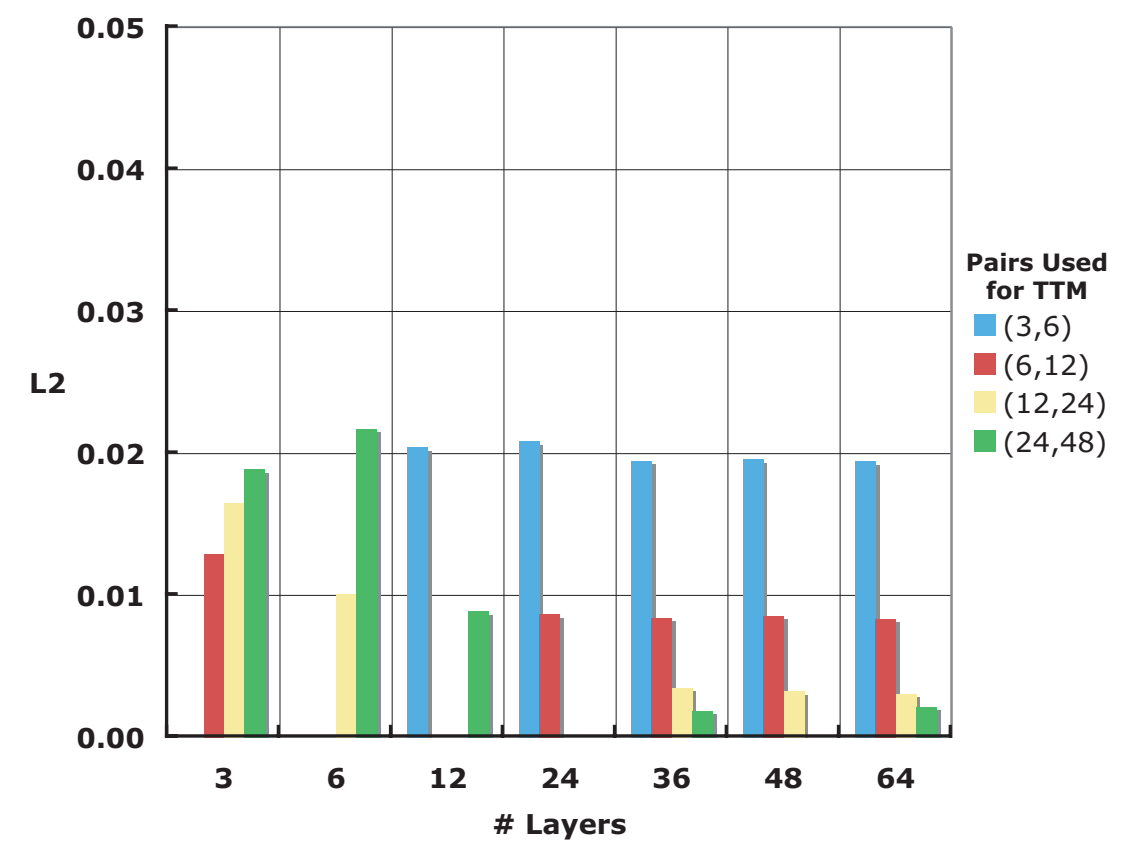

Figure 18. L2-Norm for $\left|\zeta_{m}-\zeta_{p}\right|$, for multi-layer perforate liners consisting of $10 \%$ porous perforated plates (reference OASPL of $100 \mathrm{~dB}$ ).

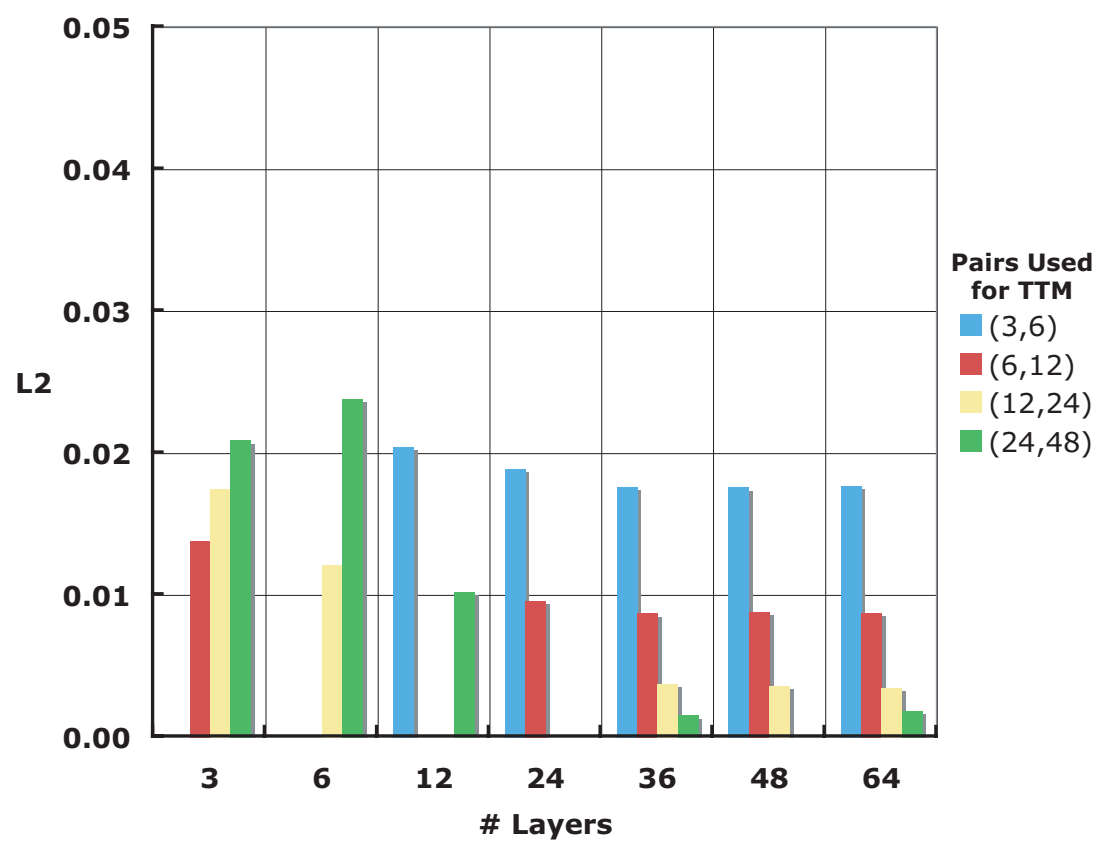

Figure 19. L2-Norm for $\left|\zeta_{m}-\zeta_{p}\right|$, for multi-layer perforate liners consisting of $10 \%$ porous perforated plates (reference OASPL of $140 \mathrm{~dB}$ ). 


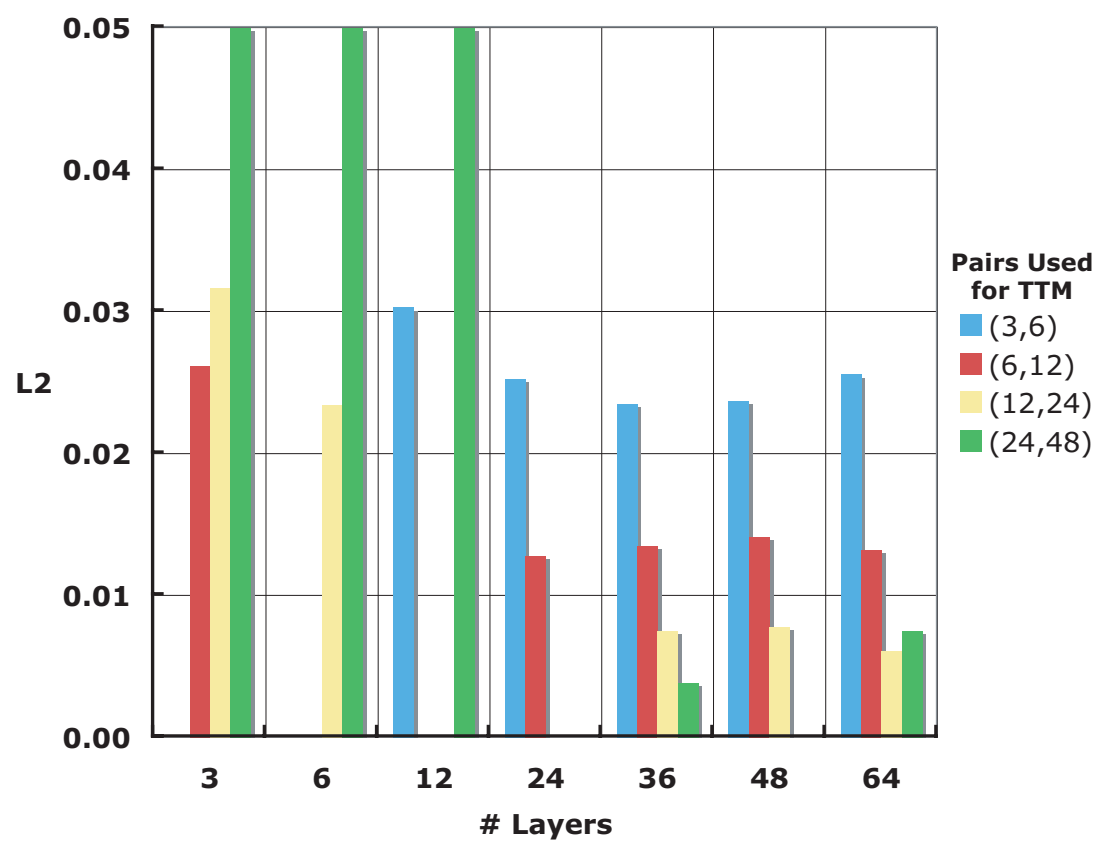

Figure 20. L2-Norm for $\left|\zeta_{m}-\zeta_{p}\right|$, for multi-layer perforate liners consisting of $5 \%$ porous perforated plates (reference OASPL of $140 \mathrm{~dB}$ ).

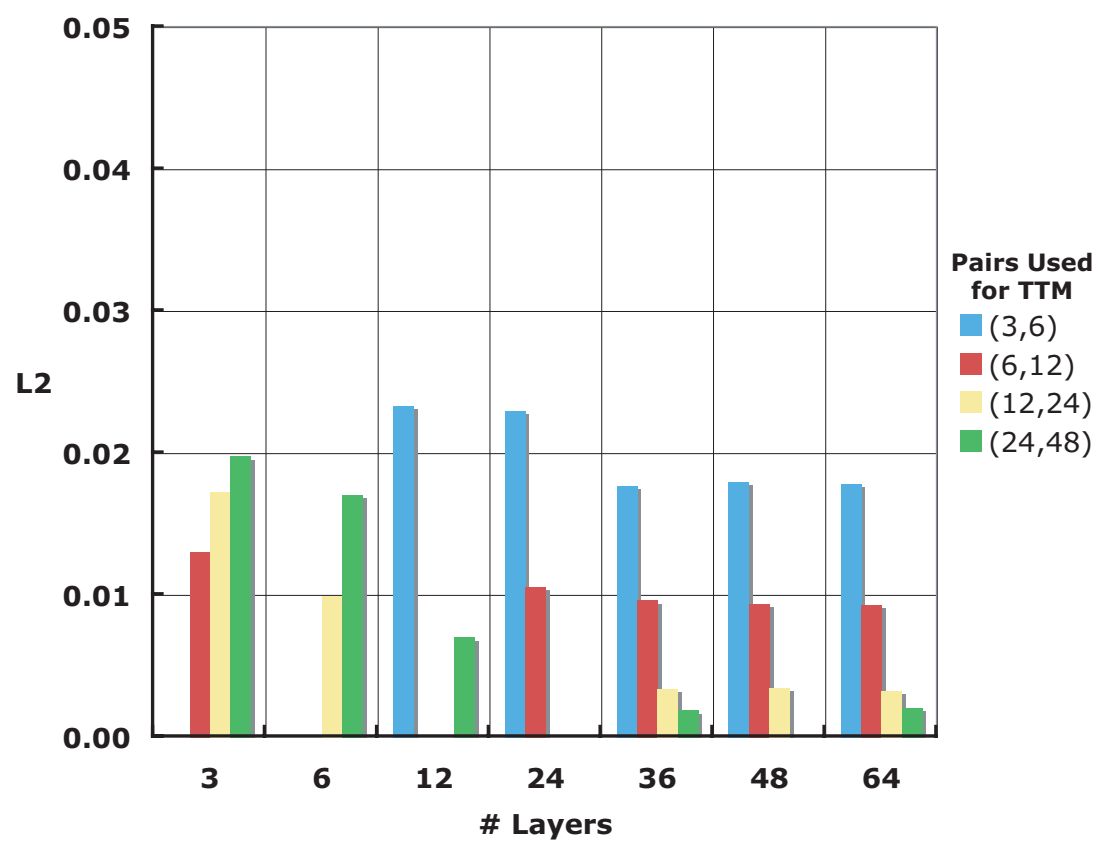

Figure 21. L2-Norm for $\left|\zeta_{m}-\zeta_{p}\right|$, for multi-layer perforate liners consisting of $15 \%$ porous perforated plates (reference OASPL of $100 \mathrm{~dB}$ ). 


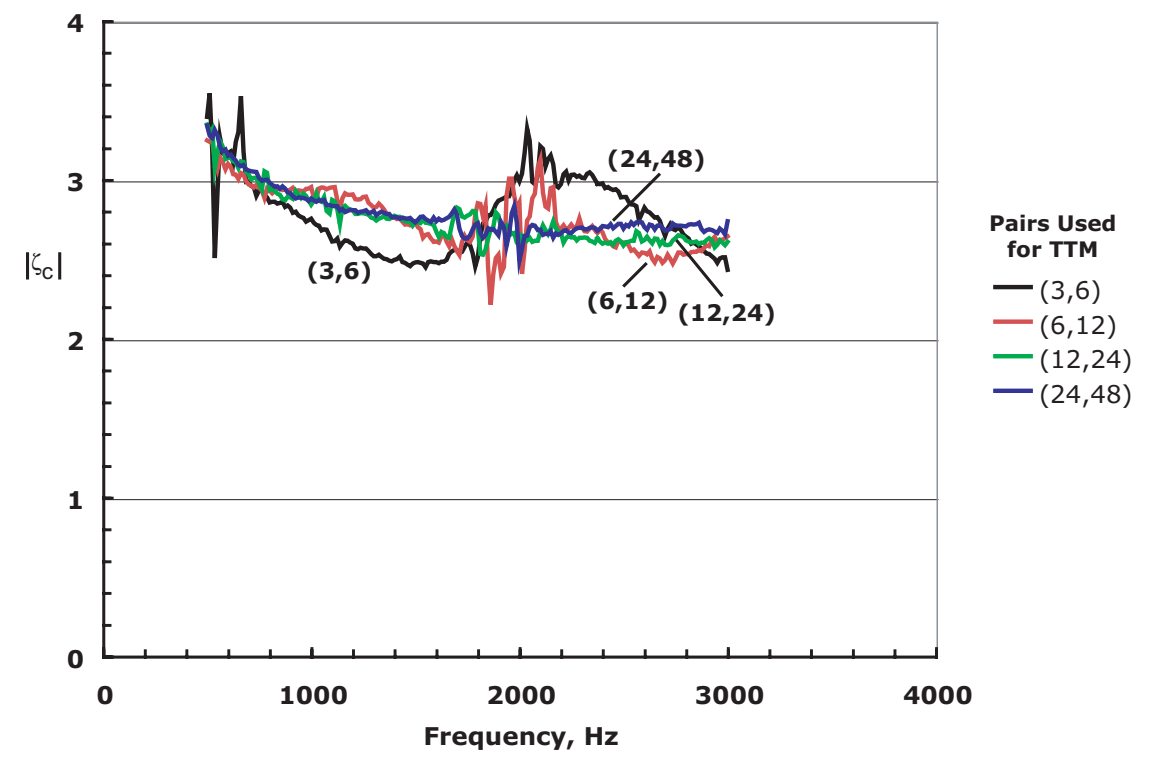

Figure 22. Characteristic impedance magnitude, $\left|\zeta_{c}\right|$, educed using four pairs of multi-layer perforate liners consisting of $5 \%$ porous perforated plates (reference OASPL of $140 \mathrm{~dB}$ ).

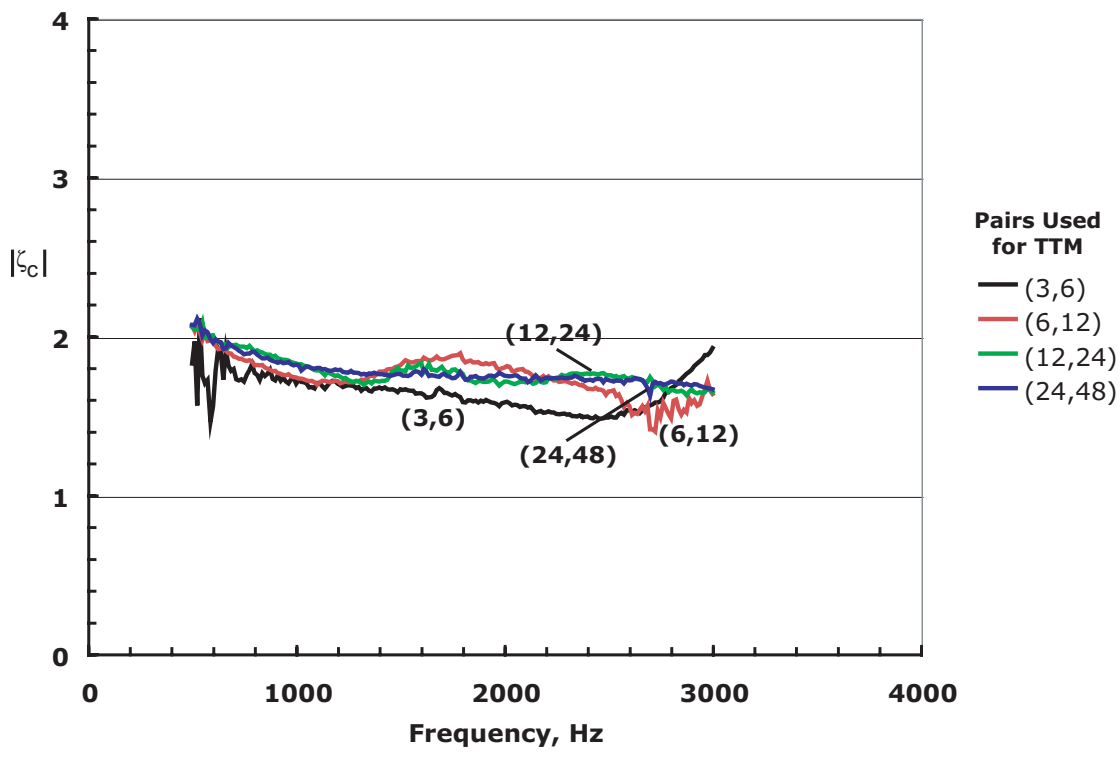

Figure 23. Characteristic impedance magnitude, $\left|\zeta_{c}\right|$, educed using four pairs of multi-layer perforate liners consisting of $15 \%$ porous perforated plates (reference OASPL of $100 \mathrm{~dB}$ ). 


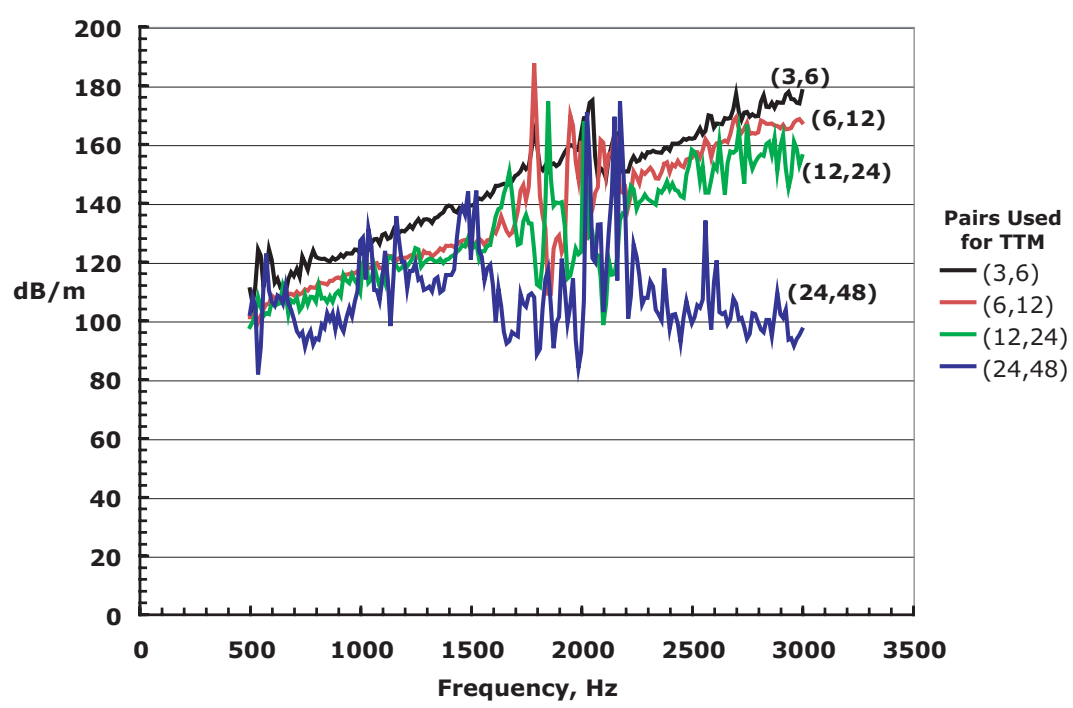

Figure 24. Attenuation rate educed using four pairs of multi-layer perforate liners consisting of 5\% porous perforated plates (reference OASPL of $140 \mathrm{~dB}$ ).

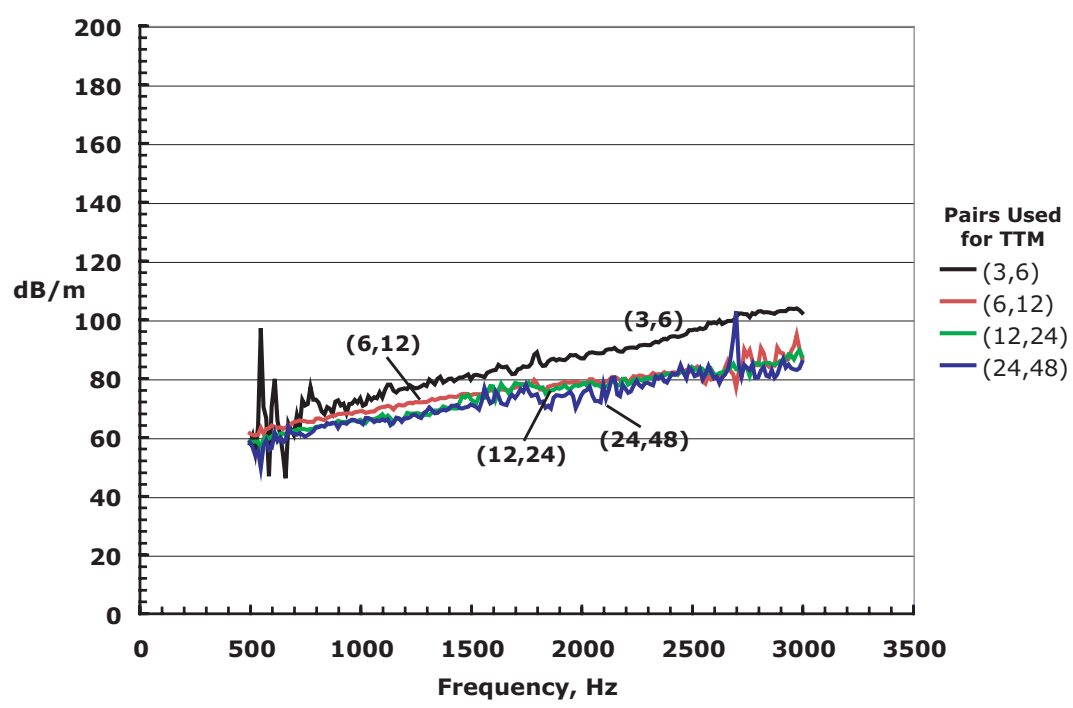

Figure 25. Attenuation rate educed using four pairs of multi-layer perforate liners consisting of 15\% porous perforated plates (reference OASPL of $100 \mathrm{~dB})$. 


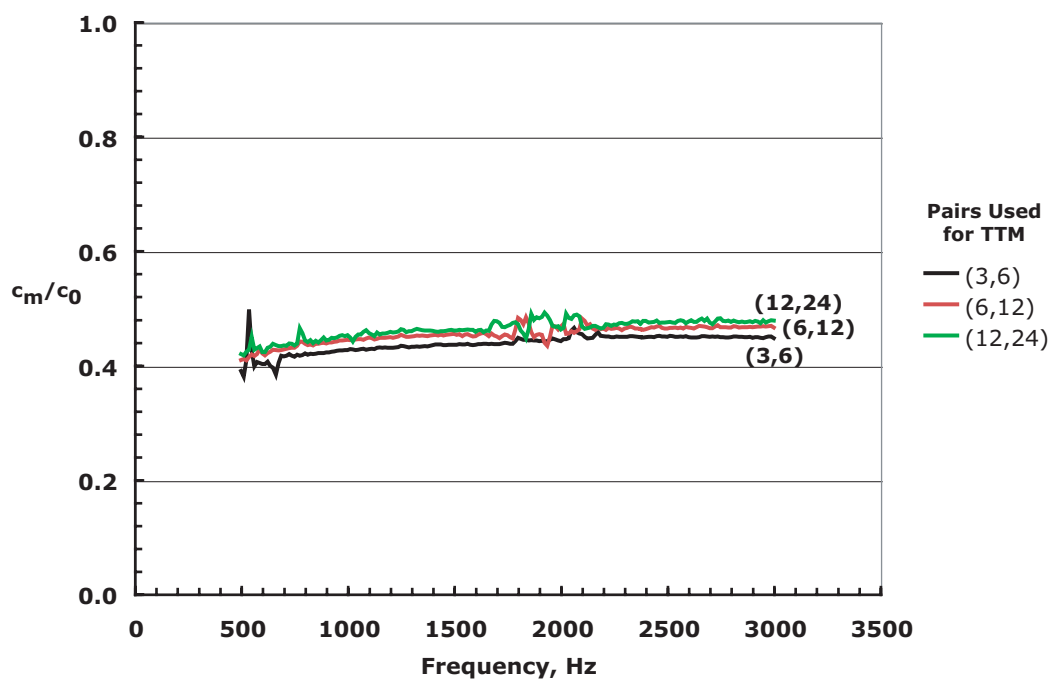

Figure 26. Ratio of sound speed to that of air, educed using three pairs of multi-layer perforate liners consisting of $5 \%$ porous perforated plates (reference OASPL of $140 \mathrm{~dB}$ ).

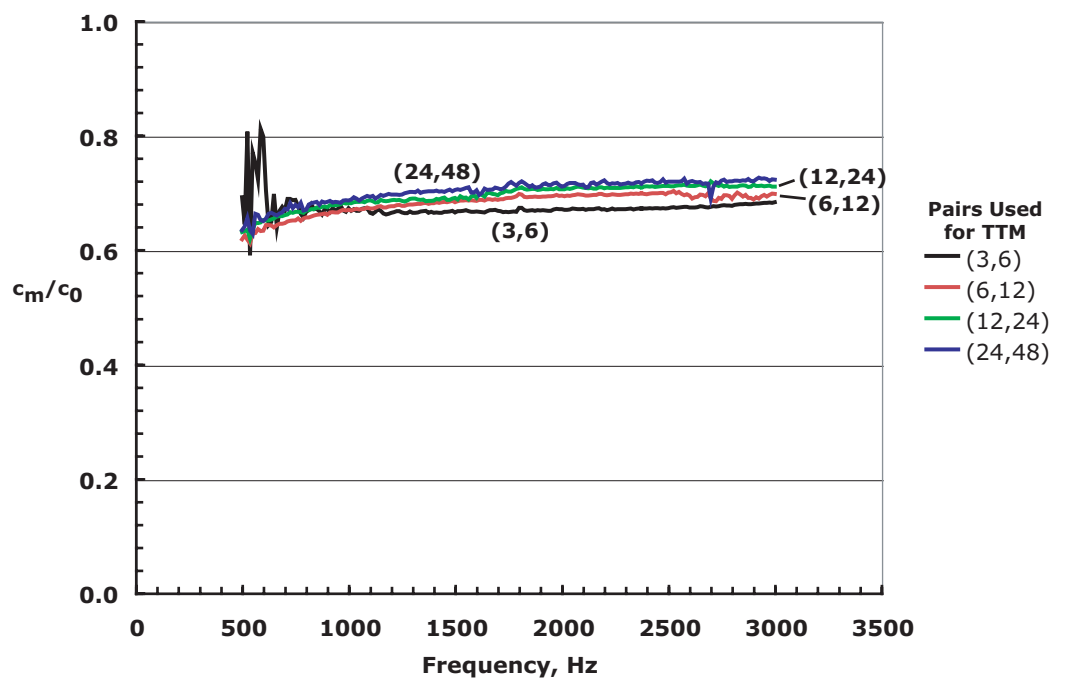

Figure 27. Ratio of sound speed to that of air, educed using four pairs of multi-layer perforate liners consisting of $15 \%$ porous perforated plates (reference OASPL of $100 \mathrm{~dB}$ ). 


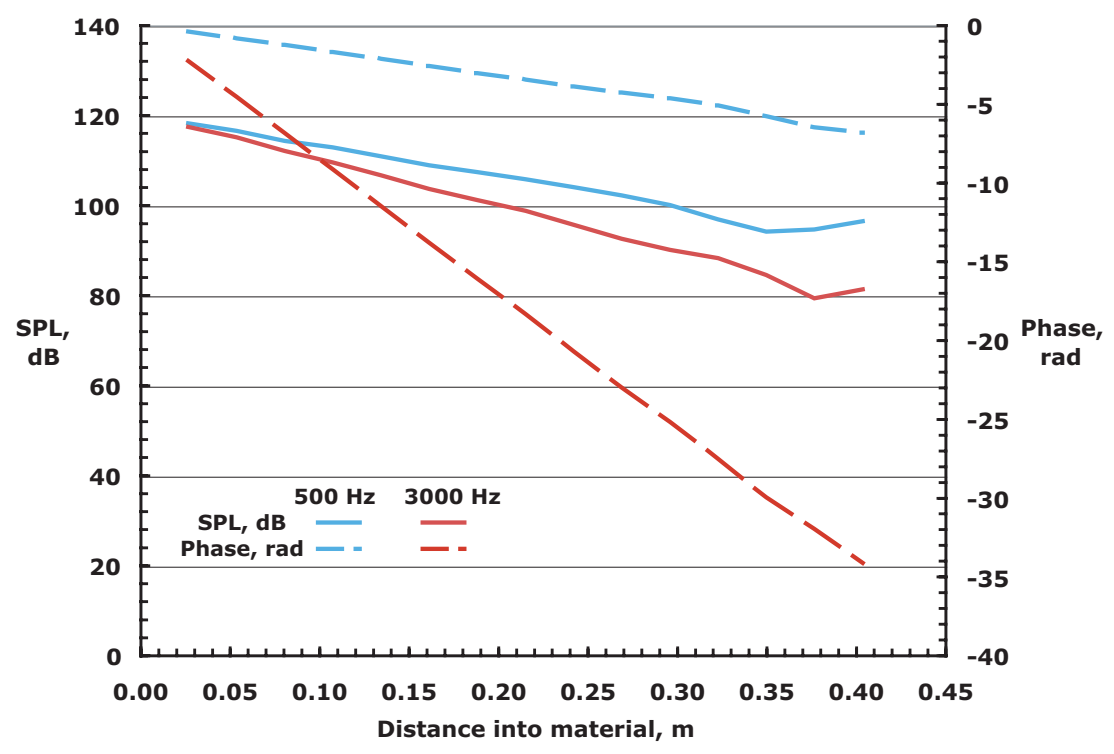

Figure 28. SPL and phase profiles used for input to Scott method, for multi-layer perforate consisting of $10 \%$ porous plates. 